\title{
Les matériaux argileux gonflants des ouvrages de fermeture de Cigéo
}

Claude Gatabin (CEA) - Frédéric Plas (Andra) - Nicolas Michau (Andra) 


\section{Plan de l'exposé}

\section{Le contexte}

- La sûreté après-fermeture de Cigéo, les différents ouvrages de fermeture de Cigéo, et leurs rôles

- Les concepts actuels d'ouvrages de fermeture de Cigéo et leurs spécifications (de l'ouvrage aux matériaux)

- Les objectifs de démonstration pour le DAC

\section{Les questionnements technologiques et scientifiques}

- La mise en œuvre industrielle des ouvrages de fermeture

- Le comportement sur le long terme

3. L'état des connaissances sur la mise en œuvre industrielle : de l'échelle du matériau à l'échelle de l'ouvrage

- Les techniques de mise en forme

- Les techniques de mise en place

4. L'état des connaissances sur les matériaux argileux gonflants : des propriétés au comportement en situations de stockage, du matériau à l'ouvrage

- Les argiles gonflantes

- Les propriétés hydrauliques et hydromécaniques non saturées et saturées

- Le comportement en situation de stockage : transfert de gaz et perturbation alcaline

\section{Conclusion (questionnements résiduels)}




\section{Schéma de principe de Cigéo}

Zone de réception, de contrôle et de préparation des colis
Zone de soutien aux travaux de creusement

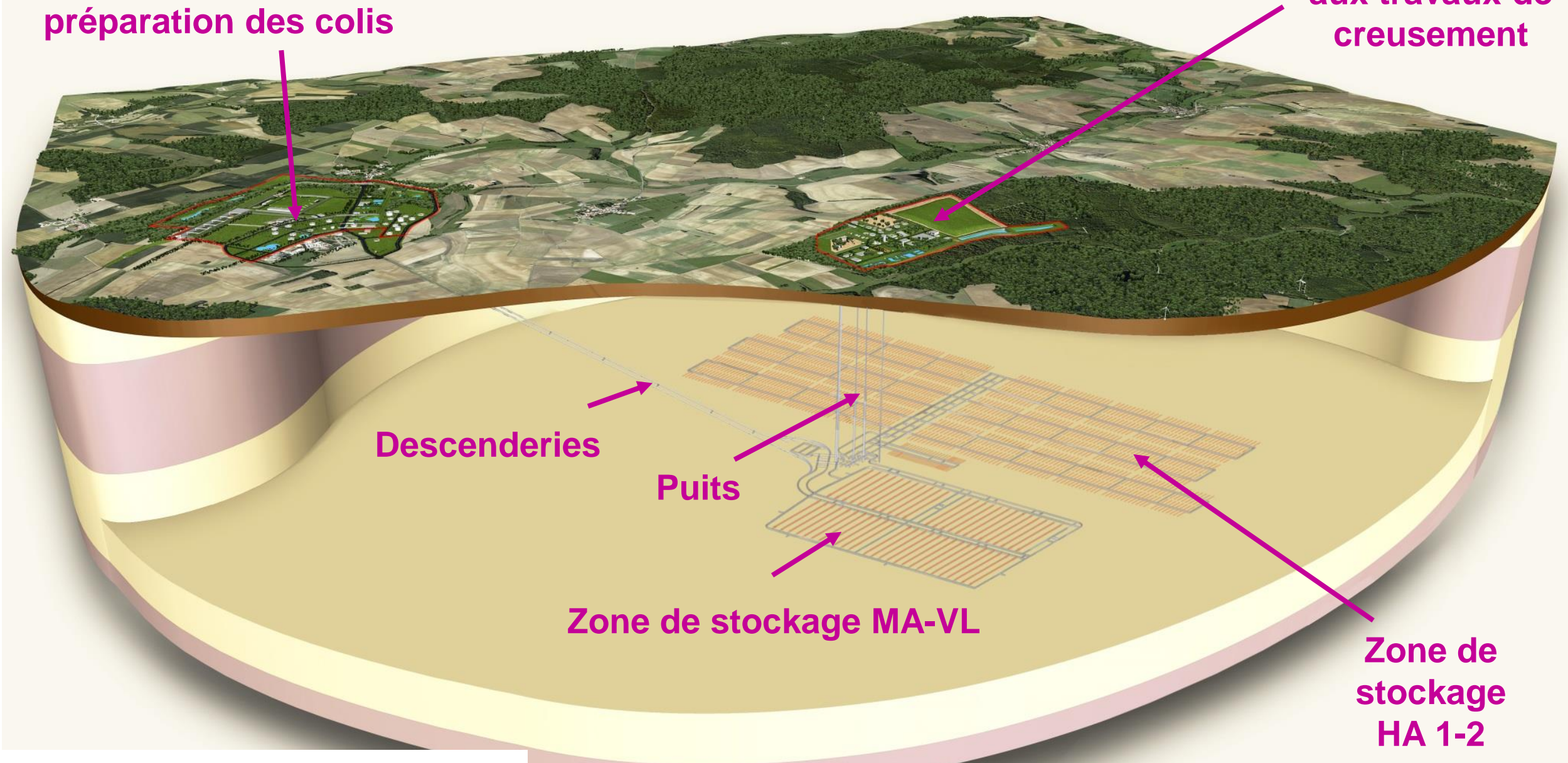

100 ans d'exploitation

Une exploitation progressive 


\section{Les ouvrages de fermeture de Cigéo}

\section{Les fonctions de sûreté en après fermeture}

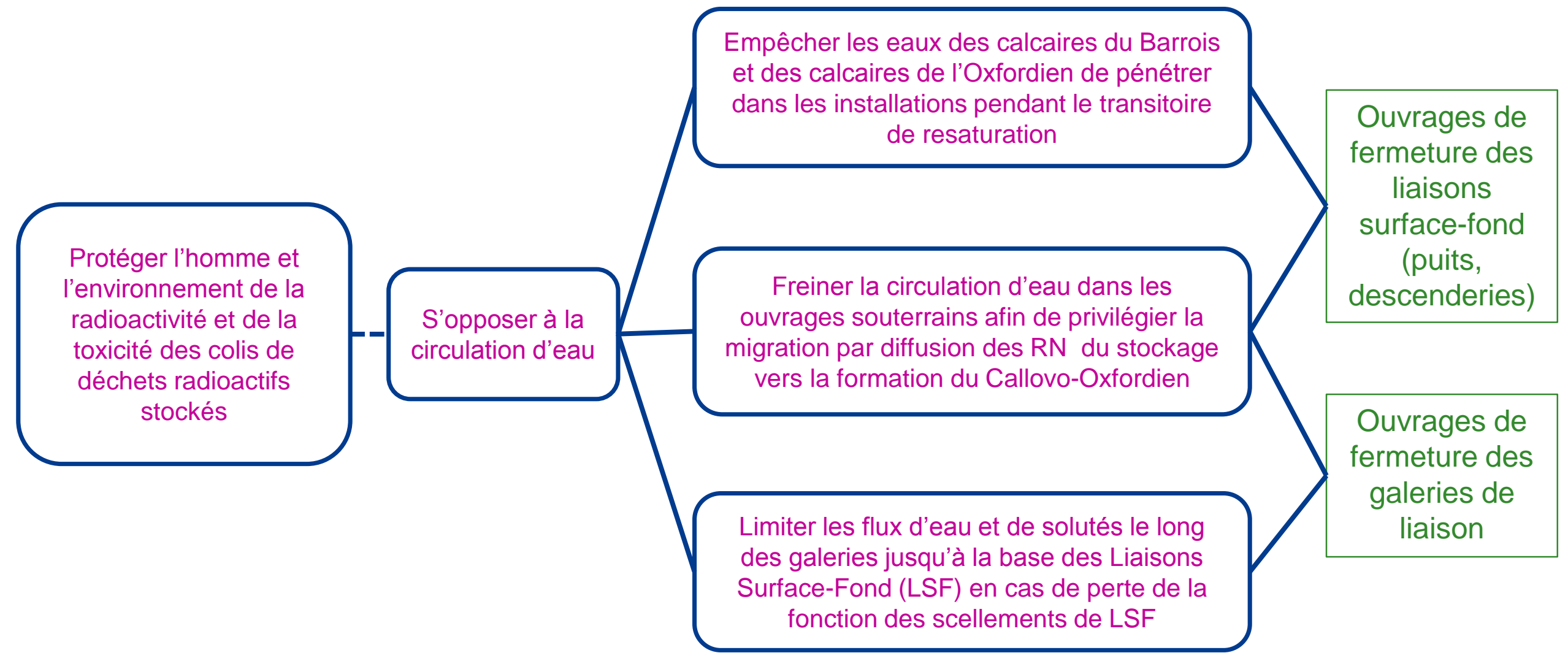

La sureté du stockage à long terme est assurée par la couche du callovo-oxfordien et les ouvrages de fermeture de liaison surface-fond 


\section{Les ouvrages de fermeture de Cigéo}

Les options techniques

L'Andra retient des objectifs de performance raisonnables et des options techniques prudentes en vue de la demande d'autorisation de création, fondés sur :

$>$ Les évaluations de sûreté,

$>$ Les connaissances acquises sur les argilites et les matériaux,

$>$ La faisabilité technologique de la construction,

$>$ La capacité de démonstration.

- Pour chaque composant du scellement

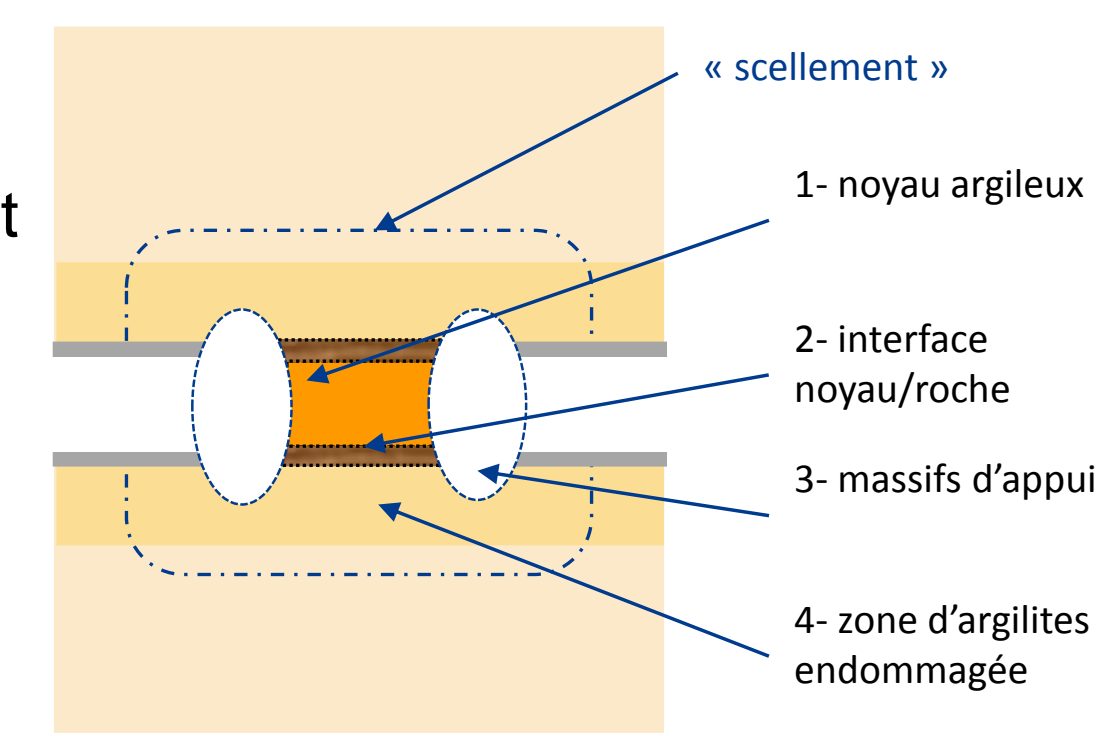

1. Noyau à base d'argile gonflante

2. Interface entre le noyau et la roche

3. Massifs d'appui

4. Zone d'argilite au droit du noyau 


\section{Les ouvrages de fermeture de Cigéo}

\section{Les schémas de principe actuels pour les liaisons surface-fond}
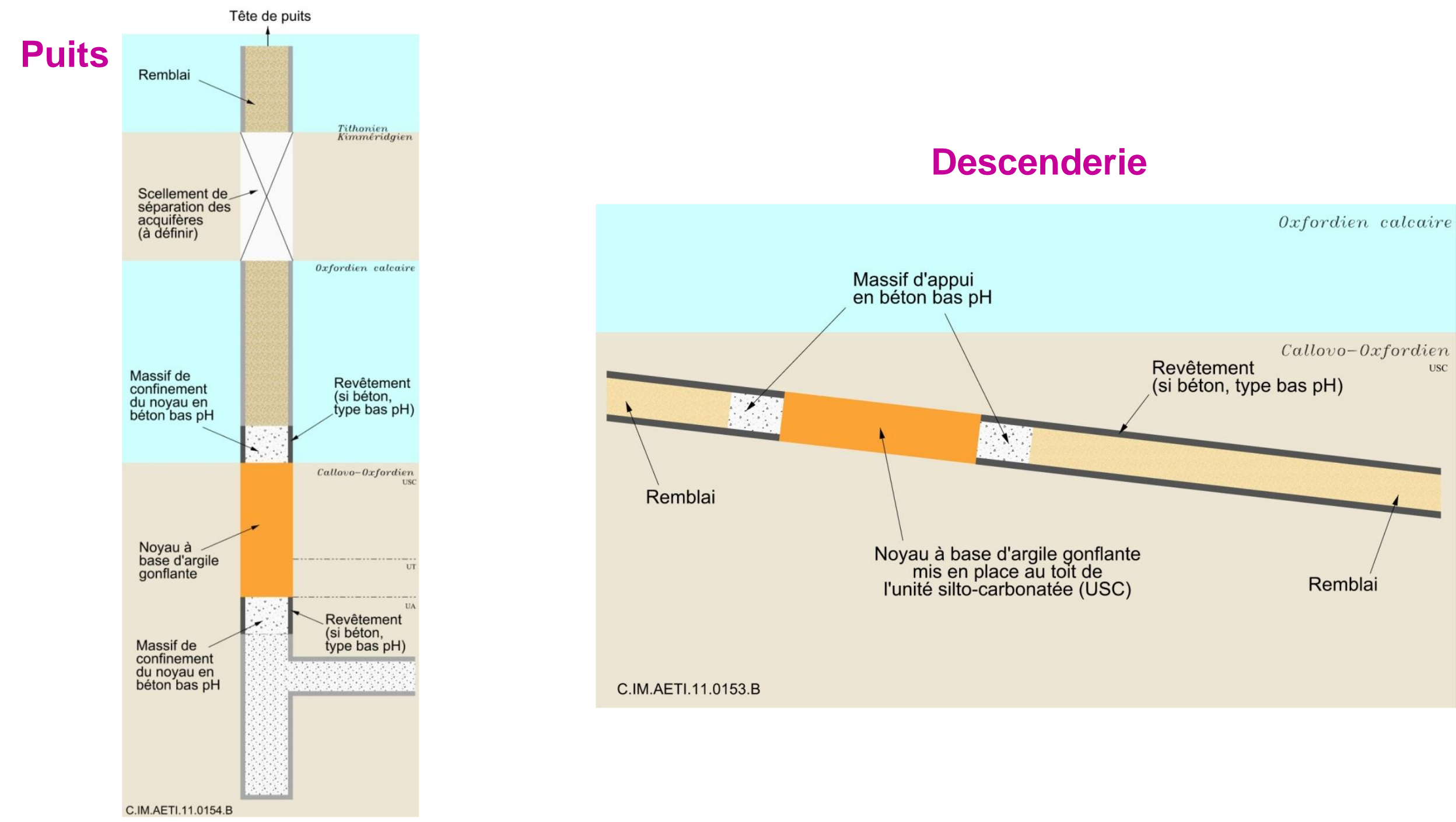


\section{Les ouvrages de fermeture de Cigéo}

Les schémas de principe actuels pour les galeries de liaison
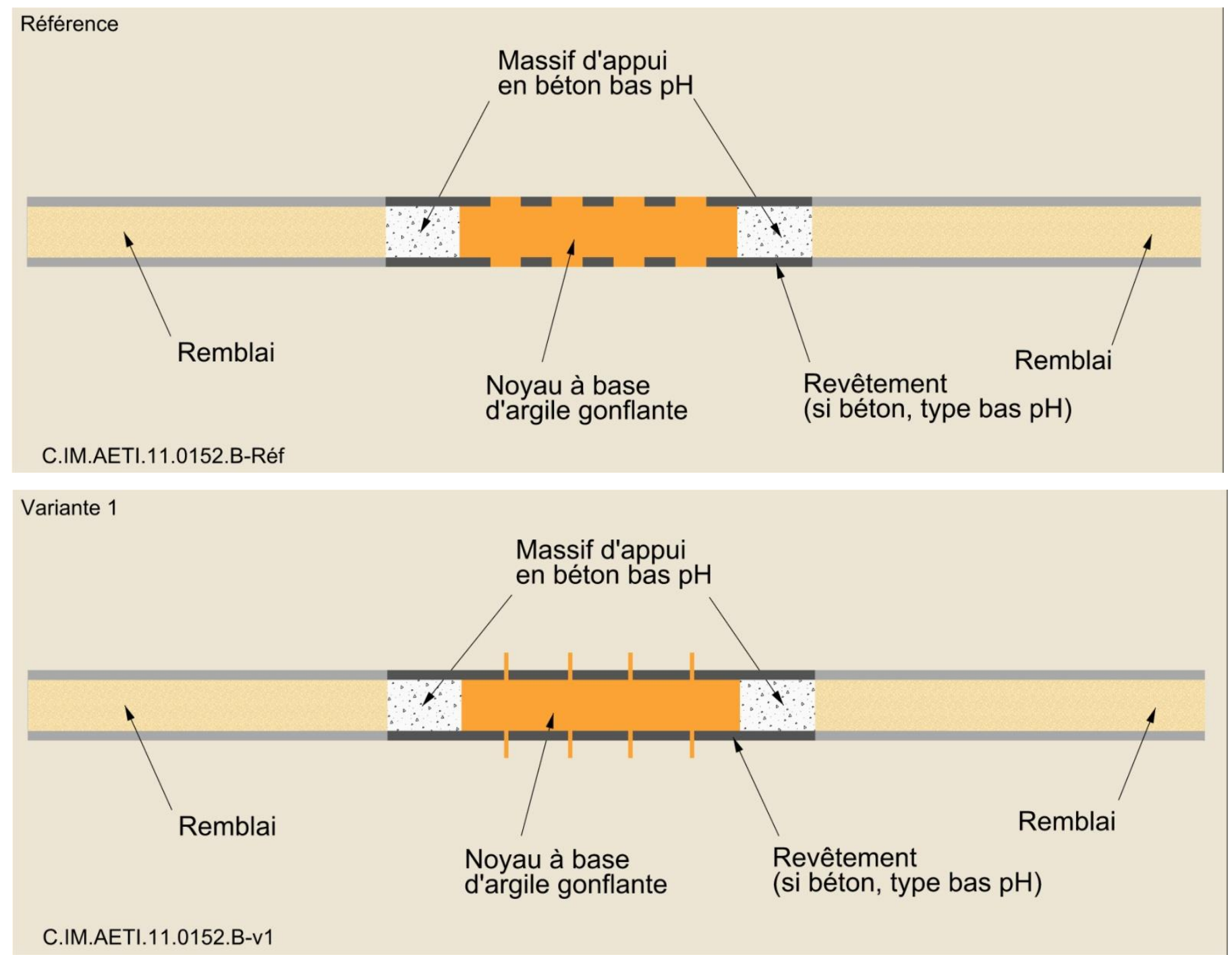


\section{Les ouvrages de fermeture de Cigéo}

Les performances des composants

$\square$ Noyau à base d'argile gonflante

$>$ Assurer la limitation du flux d'eau

$>$ Limiter les déformations du noyau et de la roche à son pourtour pour limiter l'endommagement

* Choix d'un matériau à base d'argile gonflante

$\checkmark$ Faible perméabilité $: \leq 10^{-11} \mathrm{~m} \cdot \mathrm{s}^{-1}$

$\checkmark$ Pression de gonflement

\section{$\square$ Massifs d'appui}

$>$ Garantir une saturation du noyau à volume constant

$>$ Préserver les propriétés (i.e. fonctions) des autres composants

* Choix de bétons bas pH

$\checkmark$ Faible chaleur d'hydratation, faible retrait.... 


\section{Les ouvrages de fermeture de Cigéo}

\section{L'interface noyau argileux / roche}

L'ouvrage destiné à recevoir un ouvrage de fermeture est revêtu pendant la phase d'exploitation

Quelle est la performance hydraulique de l'ensemble « noyau + revêtement »?

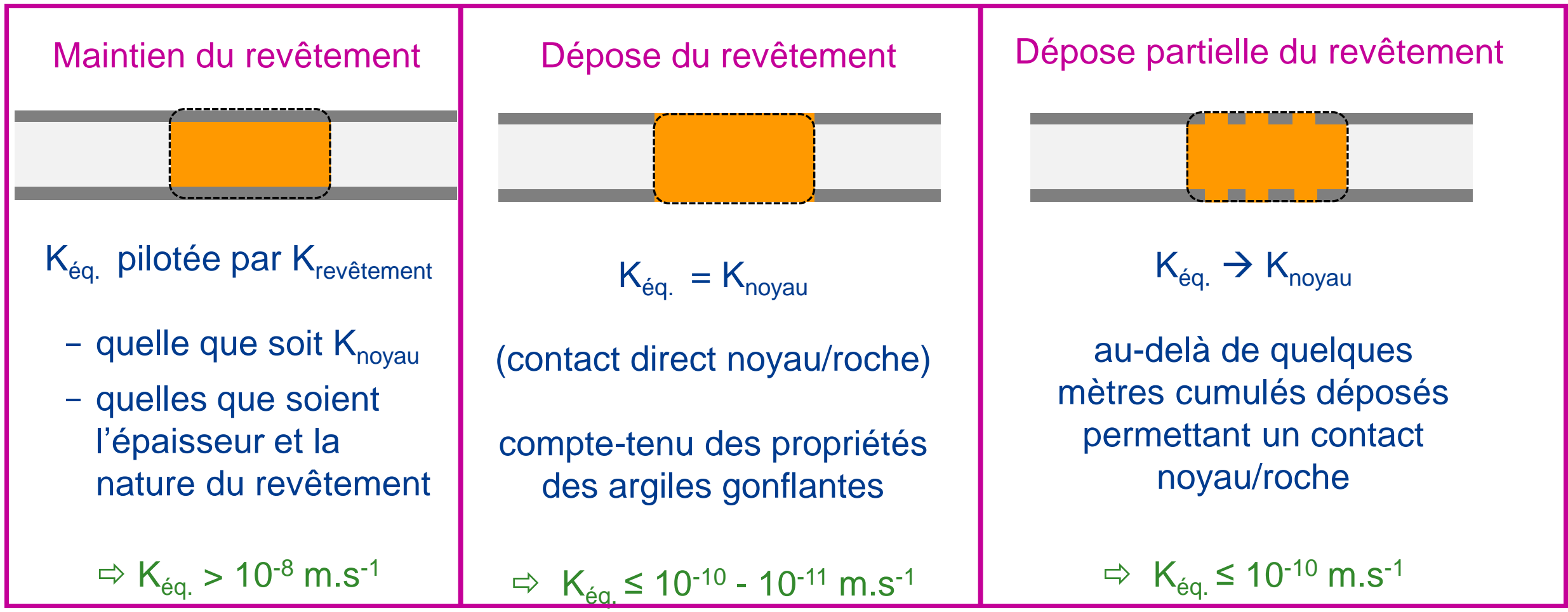

$\Rightarrow$ L'Andra retient en référence une dépose du revêtement, a minima par portions, sur une longueur cumulée de quelques mètres. 


\section{Objectifs de démonstration Le planning des études}

$\square$ Programme fortement impulsé depuis 2006

$>$ Essais en laboratoire jour et essais in situ, notamment au Laboratoire Souterrain de Bure

$>$ Maquettes et essais à échelle 1 ou à échelle représentative

$>$ Modélisation et simulations numériques

- Corpus de connaissances déjà très important pour le Dossier 2005

$\square$ Objectif : disposer des éléments de démonstration pour la DAC

$>$ Cf. Groupe permanent Déchets sur les ouvrages de fermeture en 2014

- Instruction IRSN de l'état des connaissances et du programme d'études en vue du DAC

- Avis positif de l'ASN sur la capacité de faisabilité des ouvrages de fermeture

$\square$ Construction de démonstrateurs dès la phase industrielle pilote

$>$ Disposer d'un retour d'expérience sur la période d'exploitation séculaire de Cigéo 


\section{Les questionnements technologiques et scientifiques}

\section{Les grandes questions}

\section{$\square$ Faisabilité industrielle}

> Capacité du retrait partiel du revêtement dans l'Unité Argileuse (UA) et évaluation d'un état acceptable de la zone endommagée après retrait

$>$ Conception et mise en place de l'ensemble des composants (noyau et massifs d'appui)

* Qualité des interfaces

$\checkmark$ Ouvrages de diamètre jusqu'à 9 à $10 \mathrm{~m}$ environ

$\checkmark$ Différentiation des ouvrages de fermeture : puits, descenderie, galeries

\section{$\square$ Performance hydraulique et hydromécanique}

$>$ Maitrise de la saturation en grand des noyaux des scellements

* Évaluation des modalités et des cinétiques

$>$ Maitrise du comportement HM en grand, une fois la saturation totale en eau atteinte

- Évaluation de la stabilité hydromécanique, suivant différentes sollicitations (T)HM-gaz possibles

$\checkmark$ Différentiation des ouvrages de fermeture

\section{$\square$ Evolution géochimique}

$>$ Démonstration de l'effet limité des interactions des massifs d'appui en béton sur le noyau argileux et les sur les argilites en champ proche

* Maintien des performances hydraulique et hydromécanique en grand 


\section{Les ouvrages de fermeture de Cigéo}

Mise en œuvre industrielle

\section{Le démonstrateur de scellement à échelle 1 FSS - Projet DOPAS}

Massif d'appui en béton coulé

Noyau de scellement en argile gonflante

Massif d'appui en béton projeté

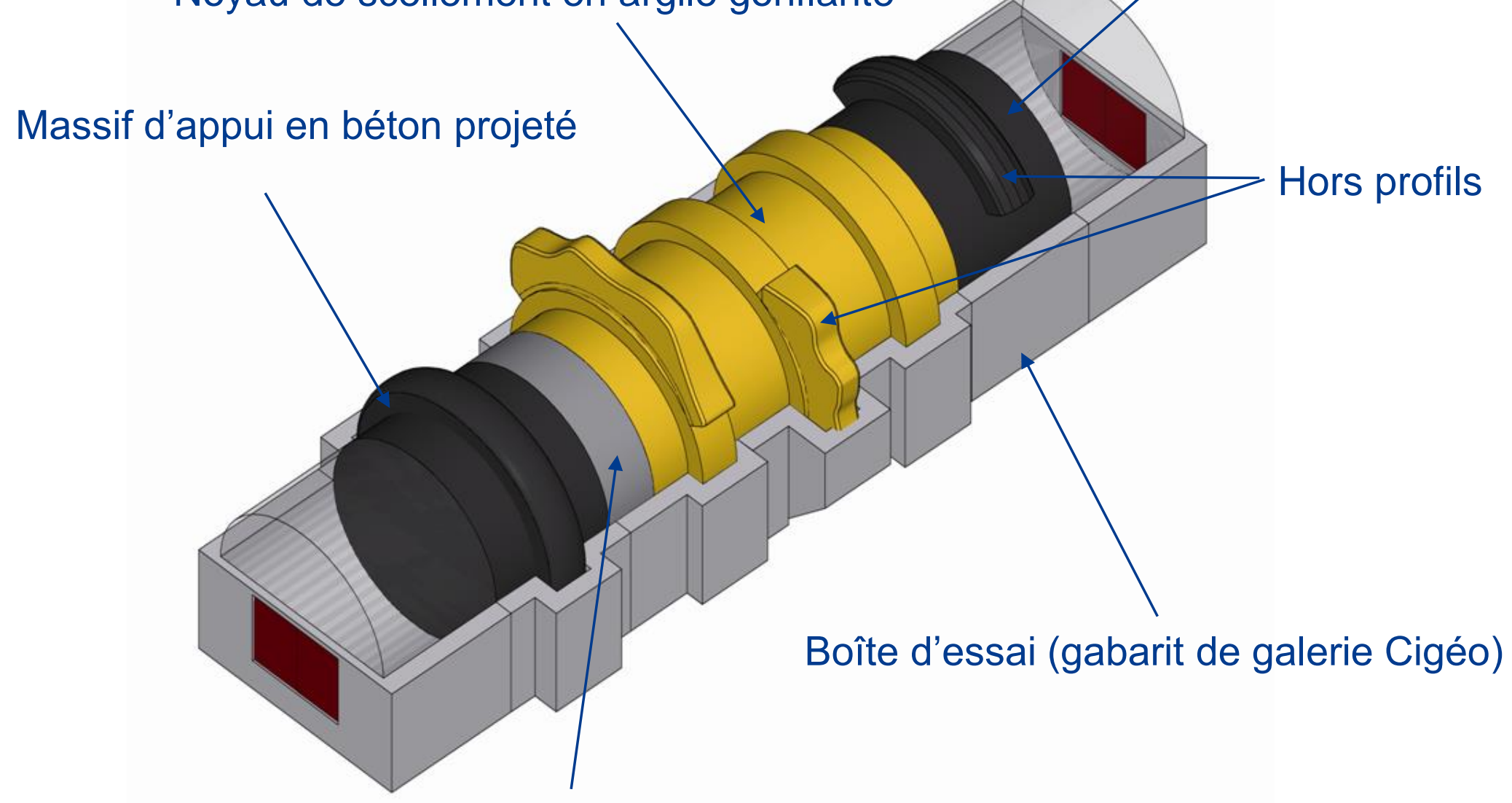

Mur de soutènement en blocs bétons préfabriqués 
Les ouvrages de fermeture de Cigéo Mise en œuvre industrielle

Le démonstrateur de scellement à échelle 1 FSS - Projet DOPAS

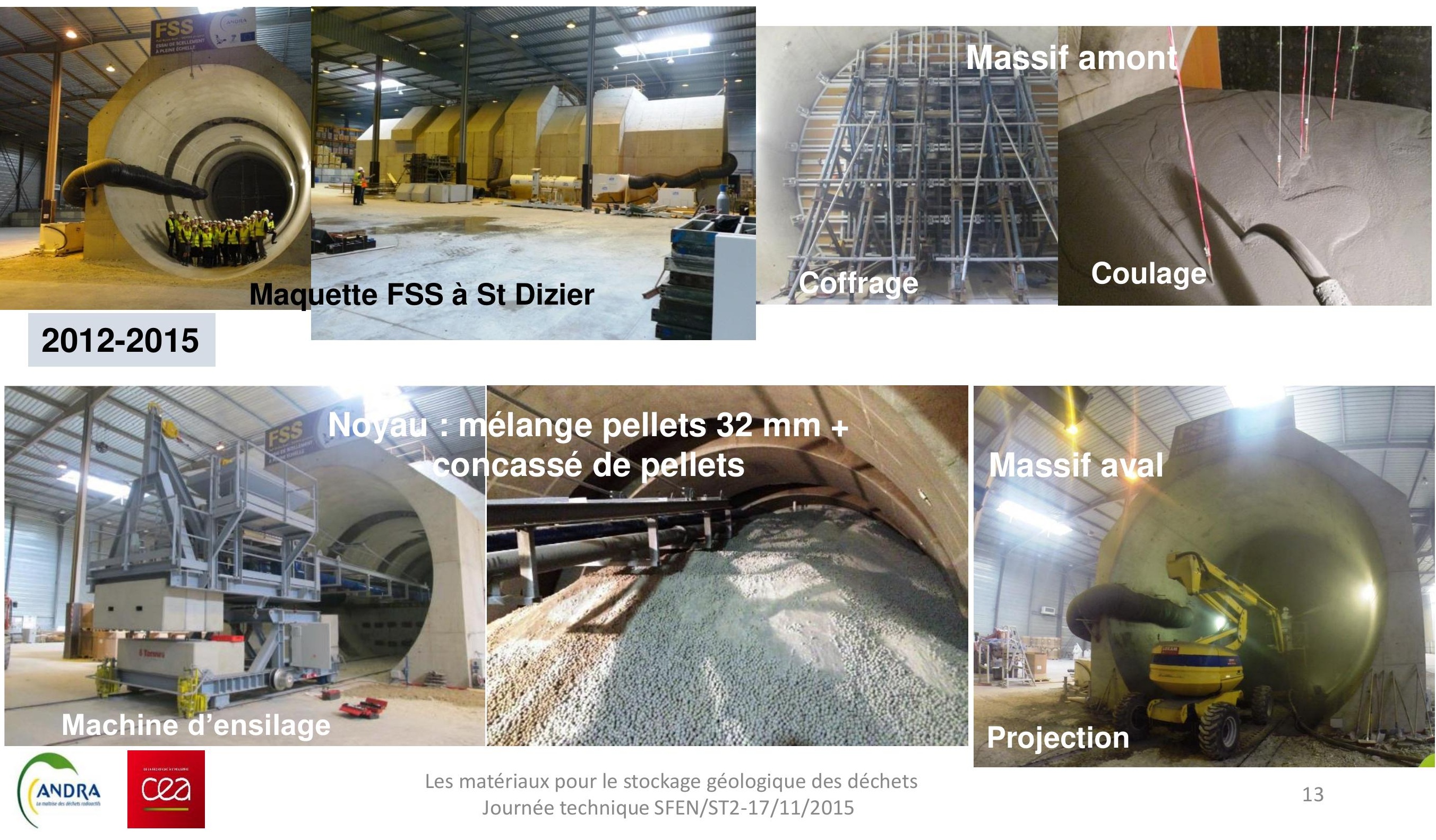




\section{Les ouvrages de fermeture de Cigéo}

\section{L'expérimentation NSC à Bure : Noyau de Scellement}

Objectif : évaluer la performance hydraulique d'un scellement en bentonite, de l'interface et de la zone endommagée en champ proche

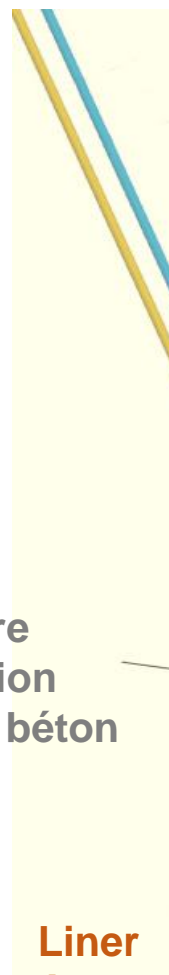

étanche
Disque

d'étanchéité

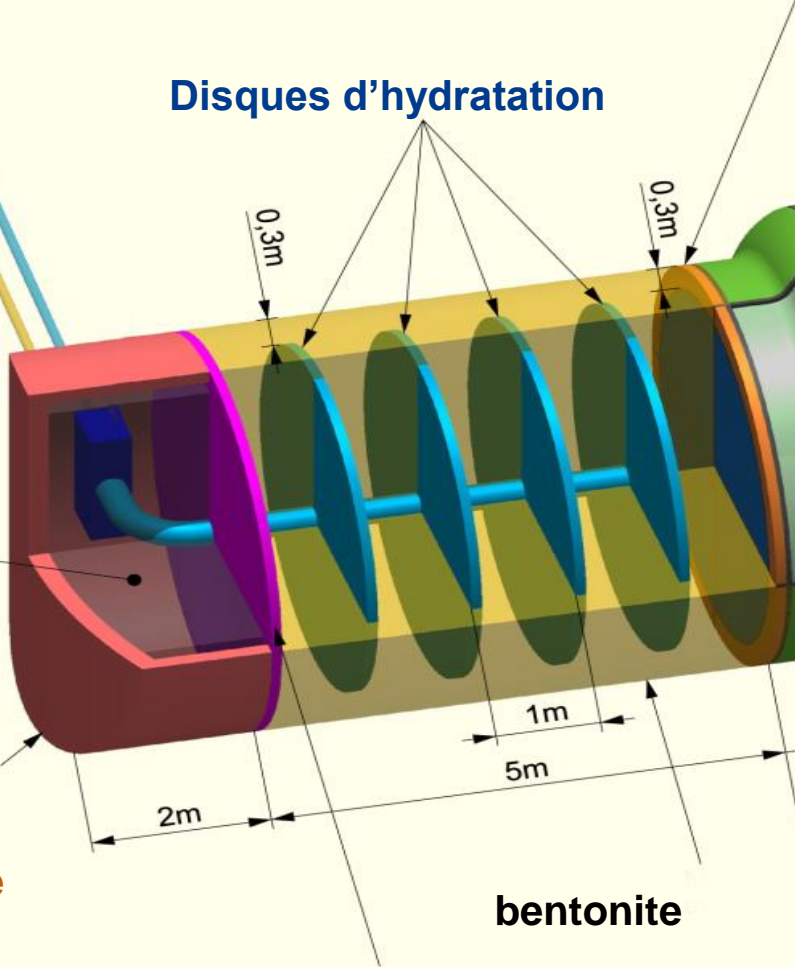

bentonite

Coupure

hydraulique

Liner étanche

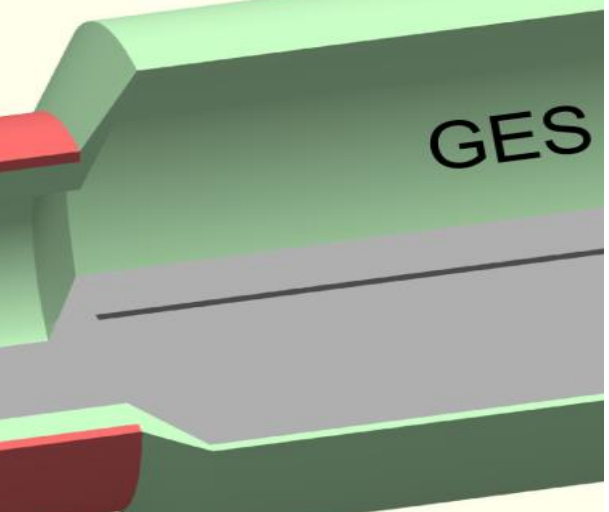




\section{Les ouvrages de fermeture de Cigéo}

\section{L'expérimentation NSC à Bure : Noyau de Scellement}

> Mélange bentonite-sable 40\% / $60 \%$

$>$ Mur: briques $300 \times 200 \times 100 \mathrm{~mm}$

$>$ Sole et vides annulaires : pellets+ poudre

$>420$ capteurs $=866$ points de mesure

Hydratation démarrée en janvier 2014

Durée prévue \pm 3 ans

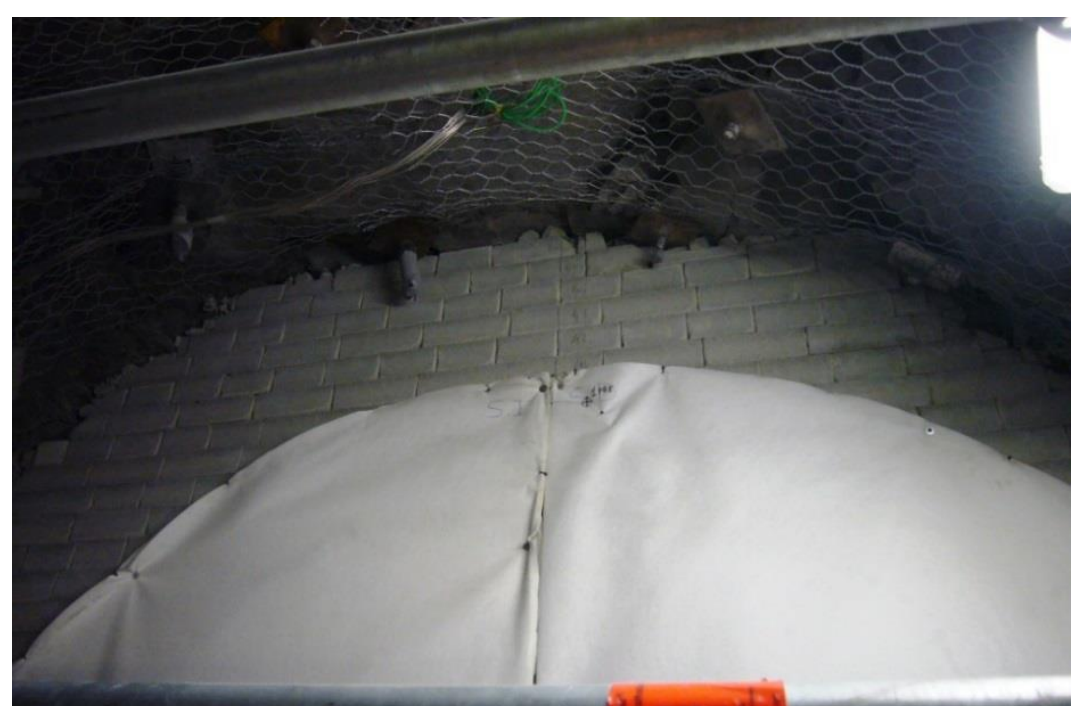

Mise en place d'un disque d'hydratation

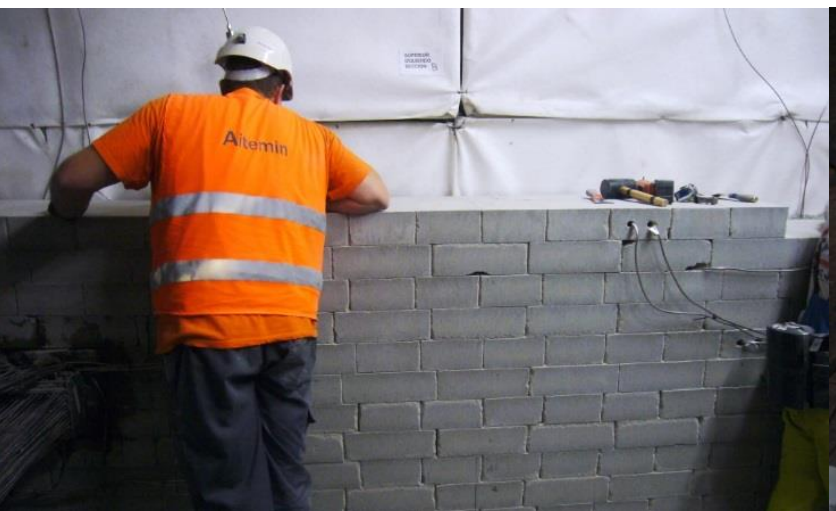

Construction du mur
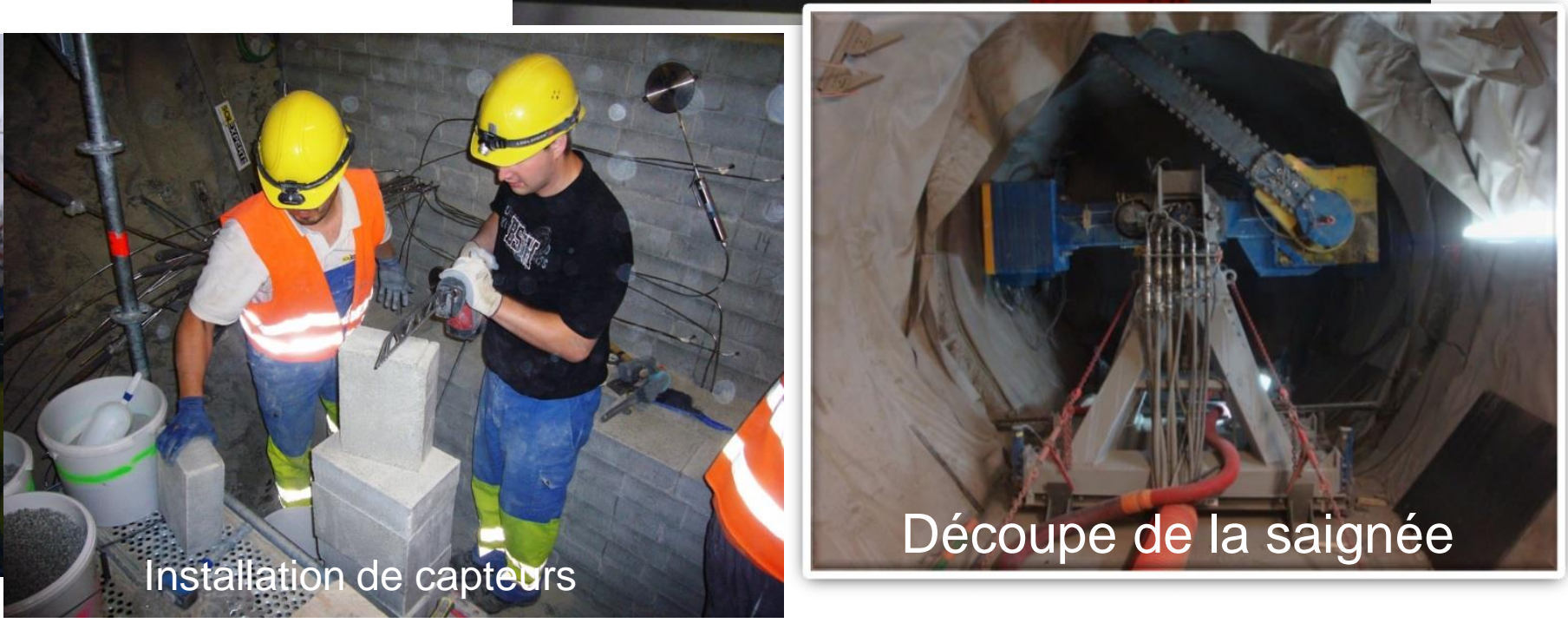


\section{Les ouvrages de fermeture de Cigéo}

\section{Les coupures hydrauliques : expérimentations TSS et SET}

Suivi de la géométrie de la saignée afin
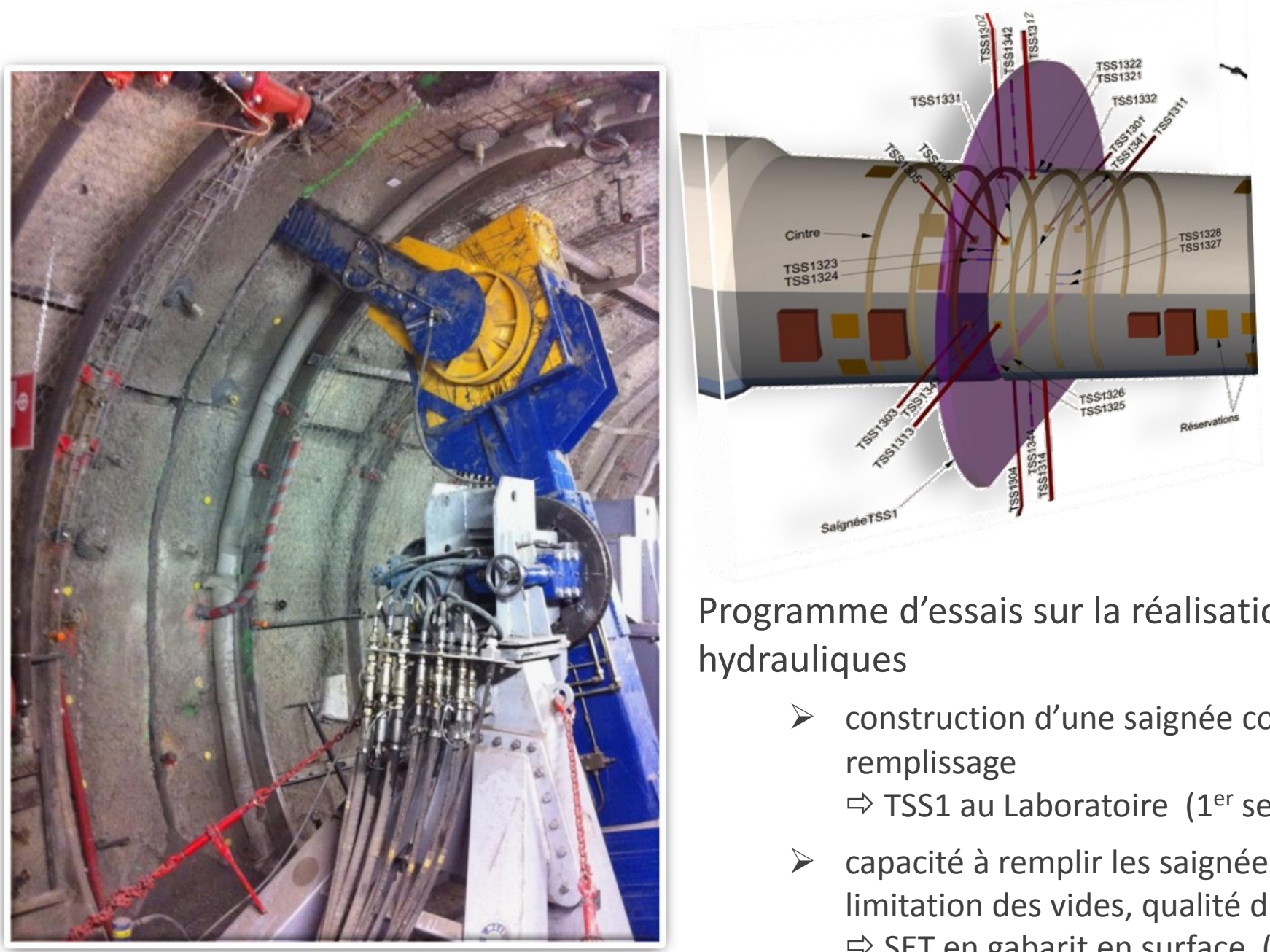
de la remplir avec une méthode industrielle (expérimentation SET à l'ETe)

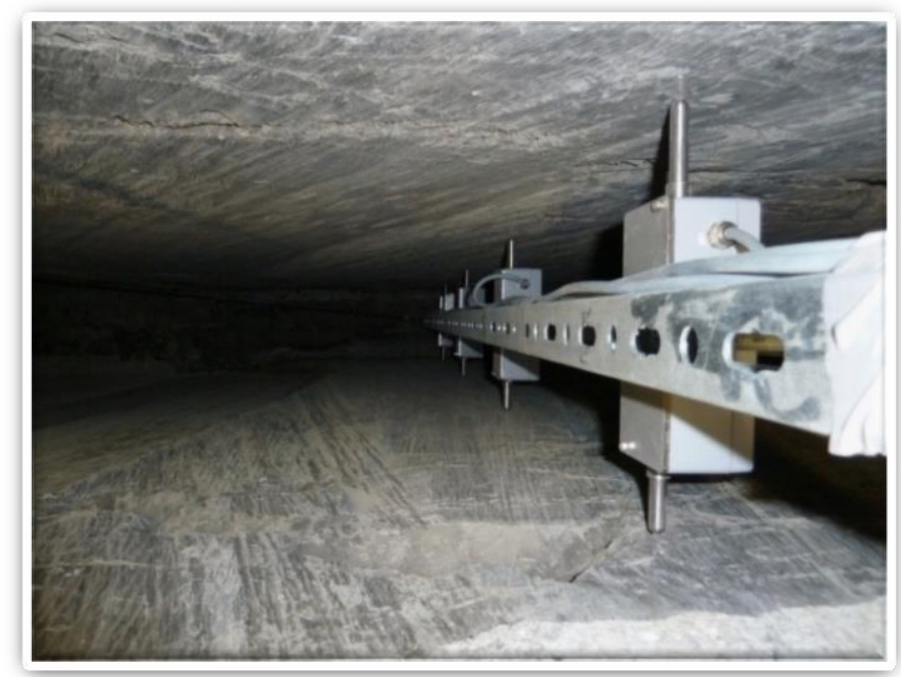

Programme d'essais sur la réalisation et la performance des coupures hydrauliques

$>$ construction d'une saignée complète $\left(360^{\circ}\right)$ et tenue des terrains avant remplissage

$\Rightarrow$ TSS1 au Laboratoire ( $1^{\text {er }}$ semestre 2011)

$>$ capacité à remplir les saignées avec de l'argile gonflante (auto-stabilité, limitation des vides, qualité du contact argile/roche) $\Rightarrow$ SET en gabarit en surface (2012-2013)

$>$ performance hydraulique de la coupure $\Rightarrow$ TSS2 au Laboratoire dans la saignée TSS1 (2013) 


\section{Les ouvrages de fermeture de Cigéo}

\section{Les coupures hydrauliques: expérimentation SET}

Etape 1 : remplissage de la partie haute par $1 / 2$ briques positionnées et coincées à l'aide d'un robot

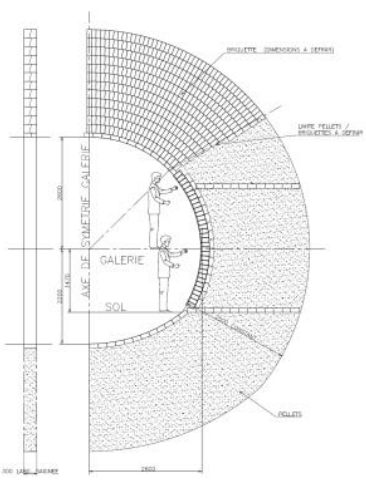

Etape 2 : Remplissage de la partie basse par un mélange pellets/poudre mis en place directement à l'aide de 2 vis sans fin
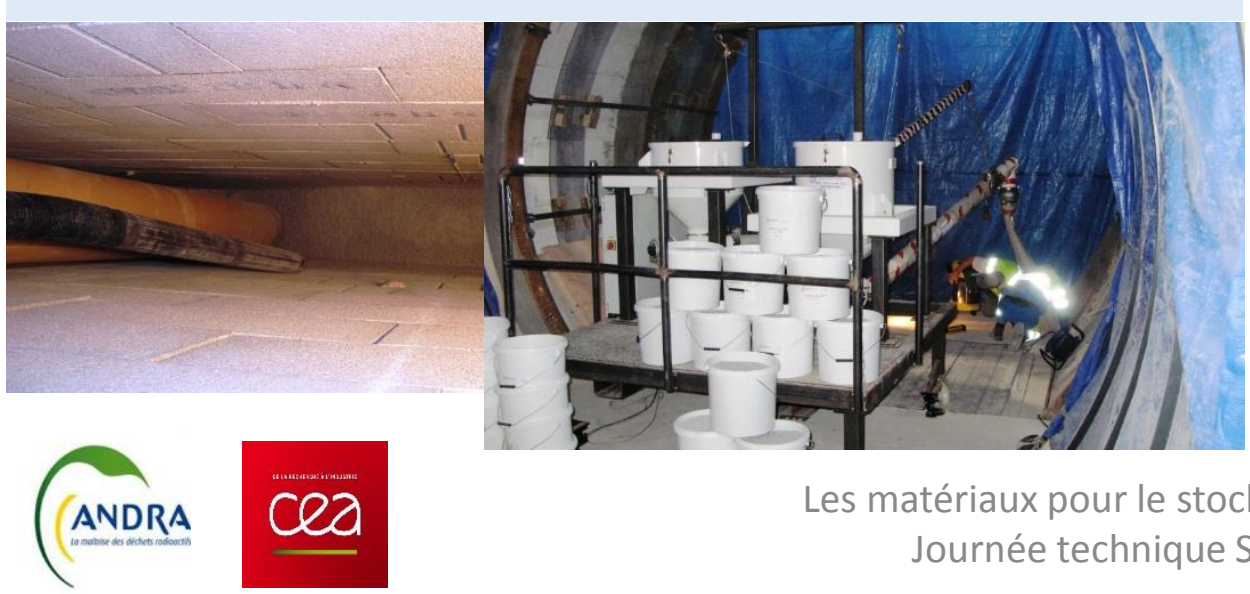

Les matériaux pour le stockag Journée technique SFE

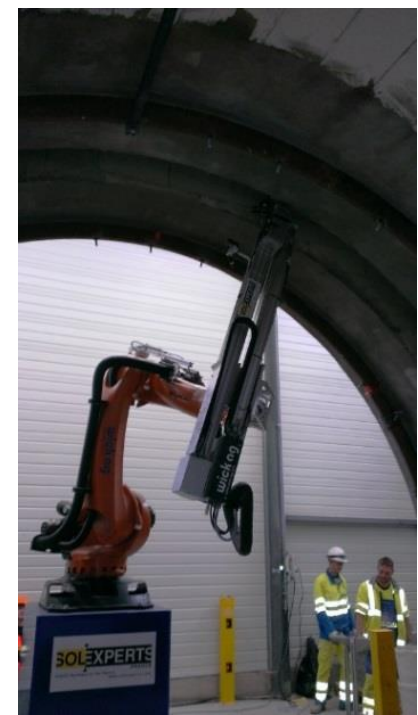

Etape 3 : fermeture de de la partie basse par $1 / 2$ briques positionnées et coincées manuellement
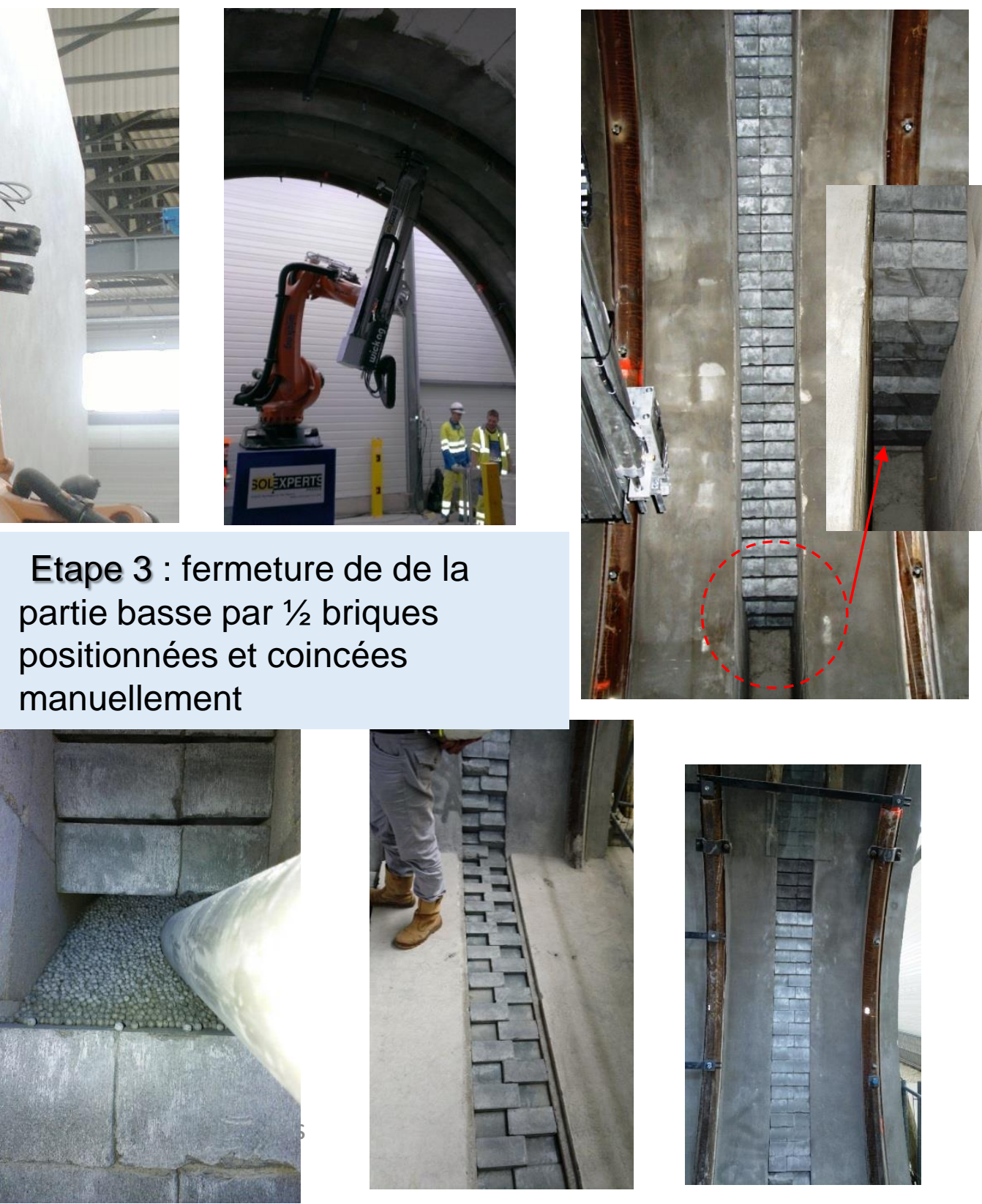


\section{Les ouvrages de fermeture de Cigéo}

L'expérimentation REM : Resaturation à l'Échelle Métrique

Objectif : réaliser la saturation d'un noyau de bentonite constitué du mélange FSS à une échelle représentative

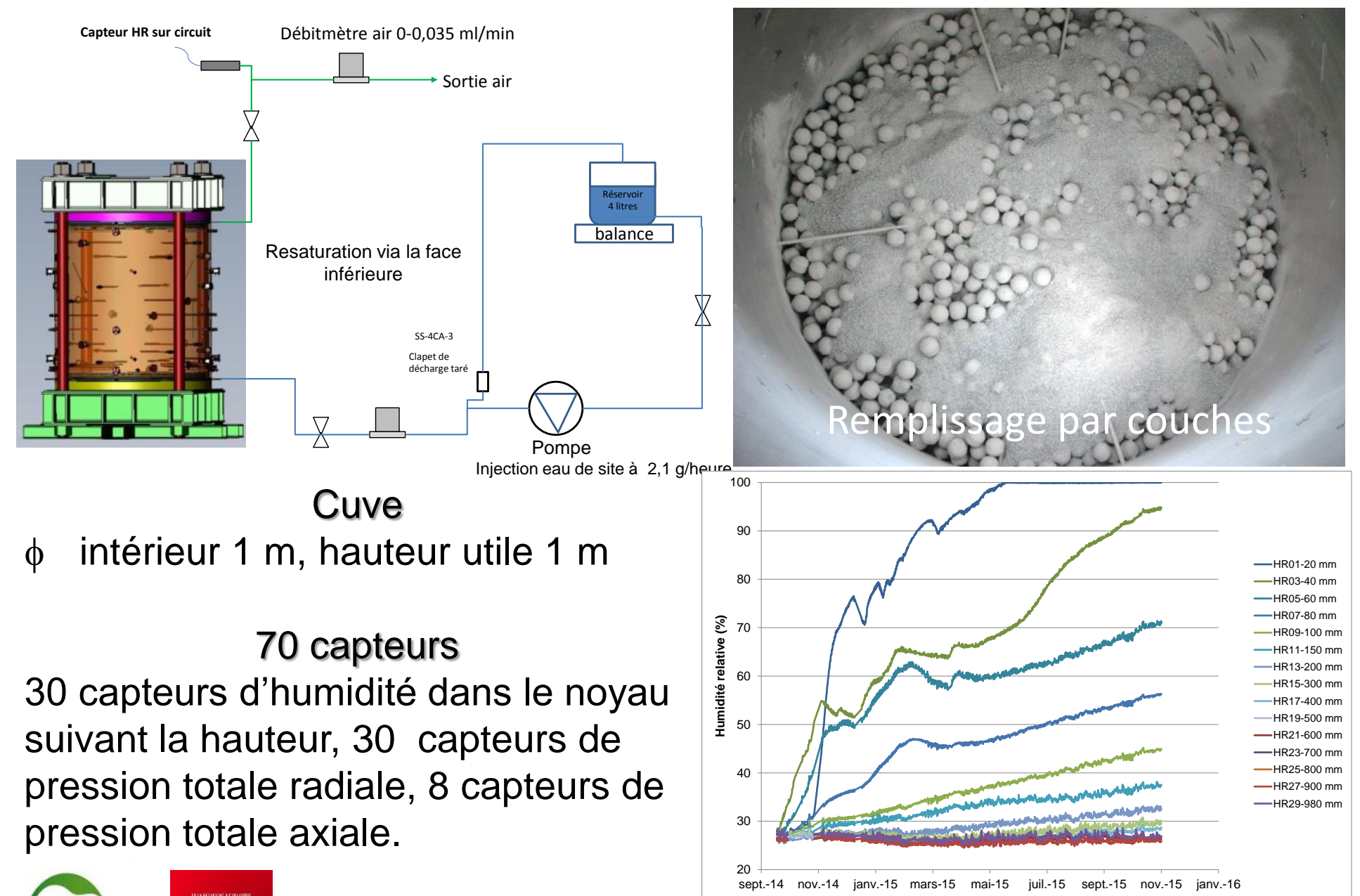

ANDRA CEA
Essai démarré le 25/09/2014 pour une durée $>10$ ans

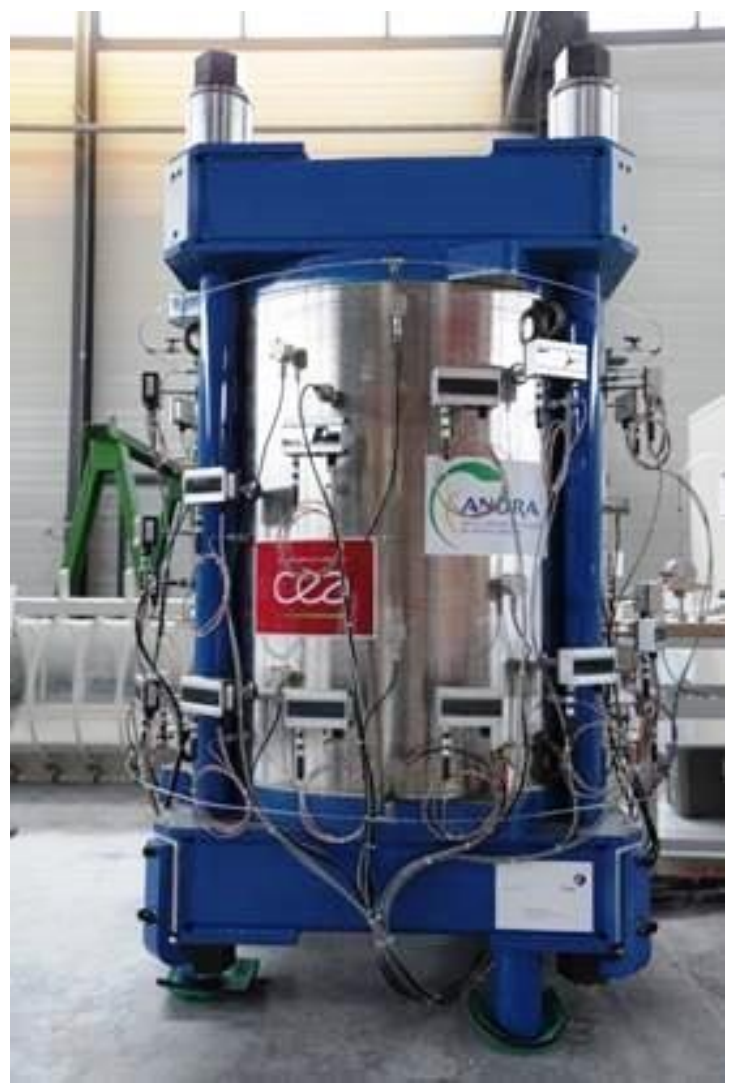




\section{Les techniques de mise en forme}

Diverses techniques de mise en forme ont été étudiées et éprouvées

Pellets

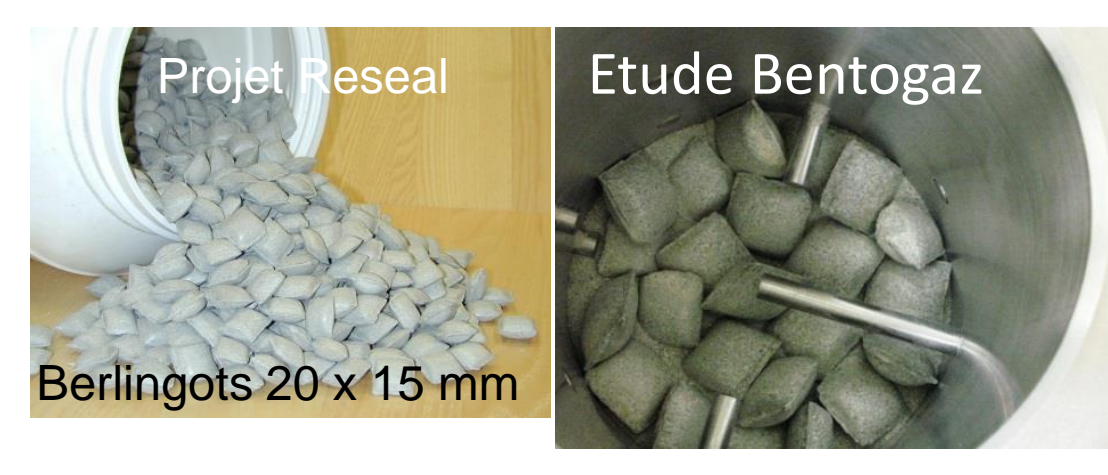

arengas

20 PGZ, Bure
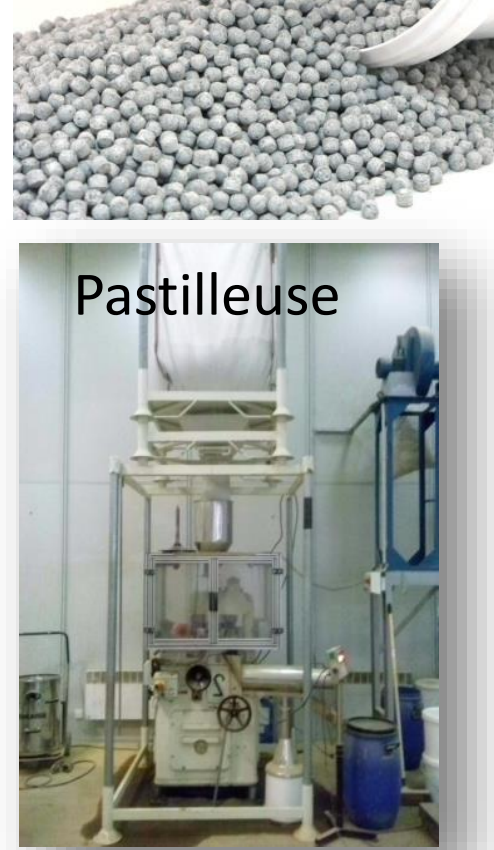
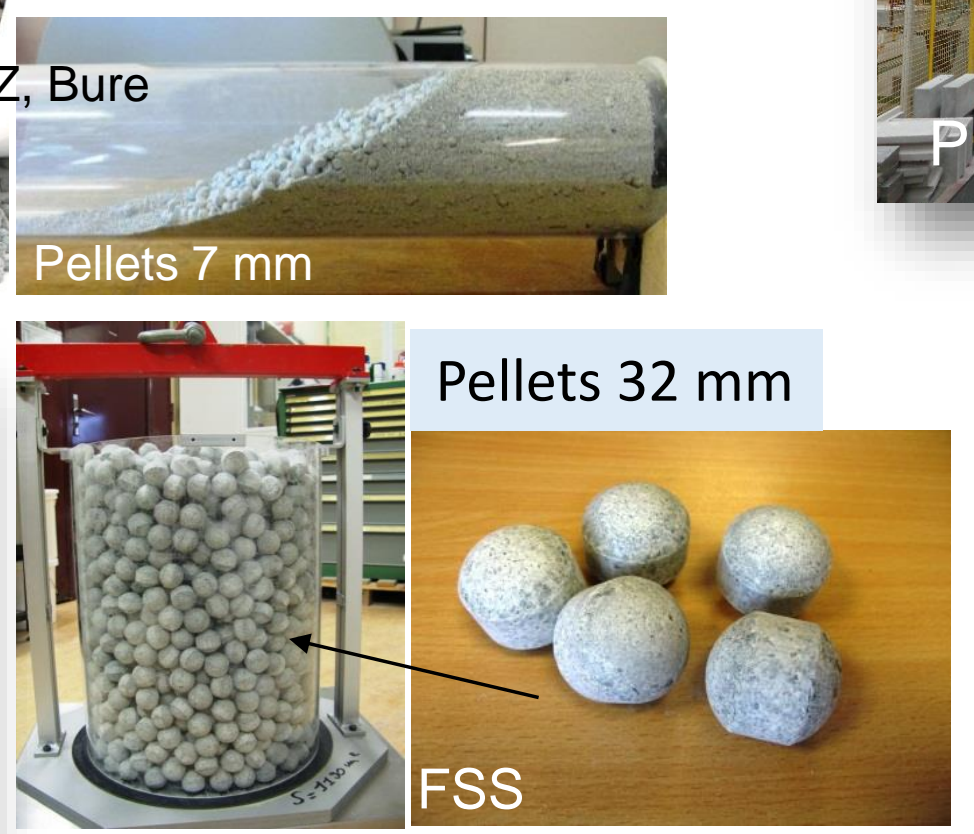

Les matériaux pour le stockage géologique des déchets Journée technique SFEN/ST2-17/11/2015
Compactage dans un moule

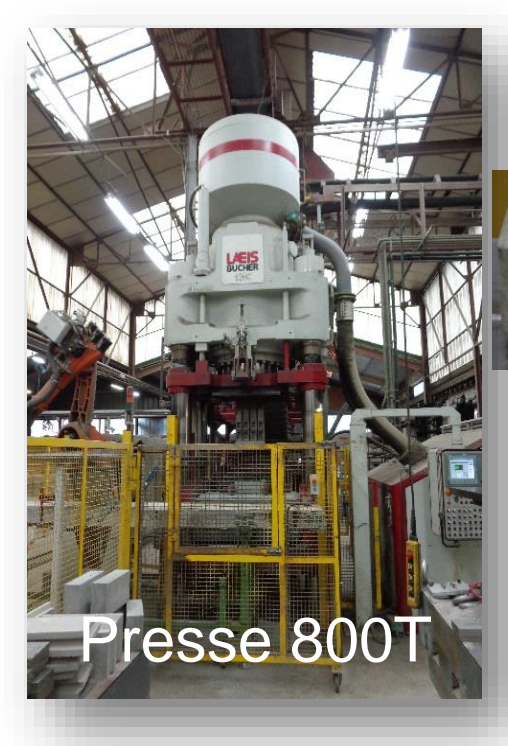

Briques

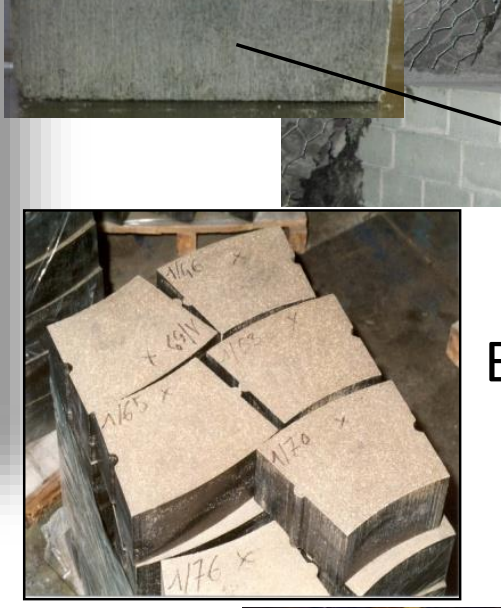

Anneaux 2,3 $\mathrm{m} \times 0,5 \mathrm{~m}$ Poids : 4 tonnes

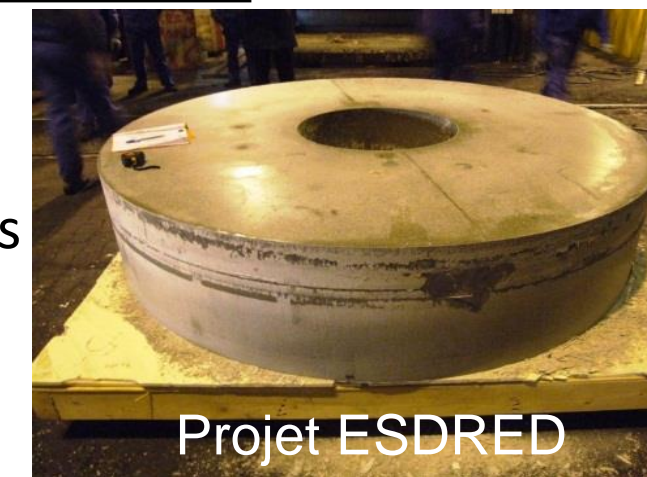




\section{Les techniques de mise en forme}

D’autre techniques sont possibles, pour traiter des concepts spécifiques ou des cas particuliers

Compactage isostatique dans un moule souple
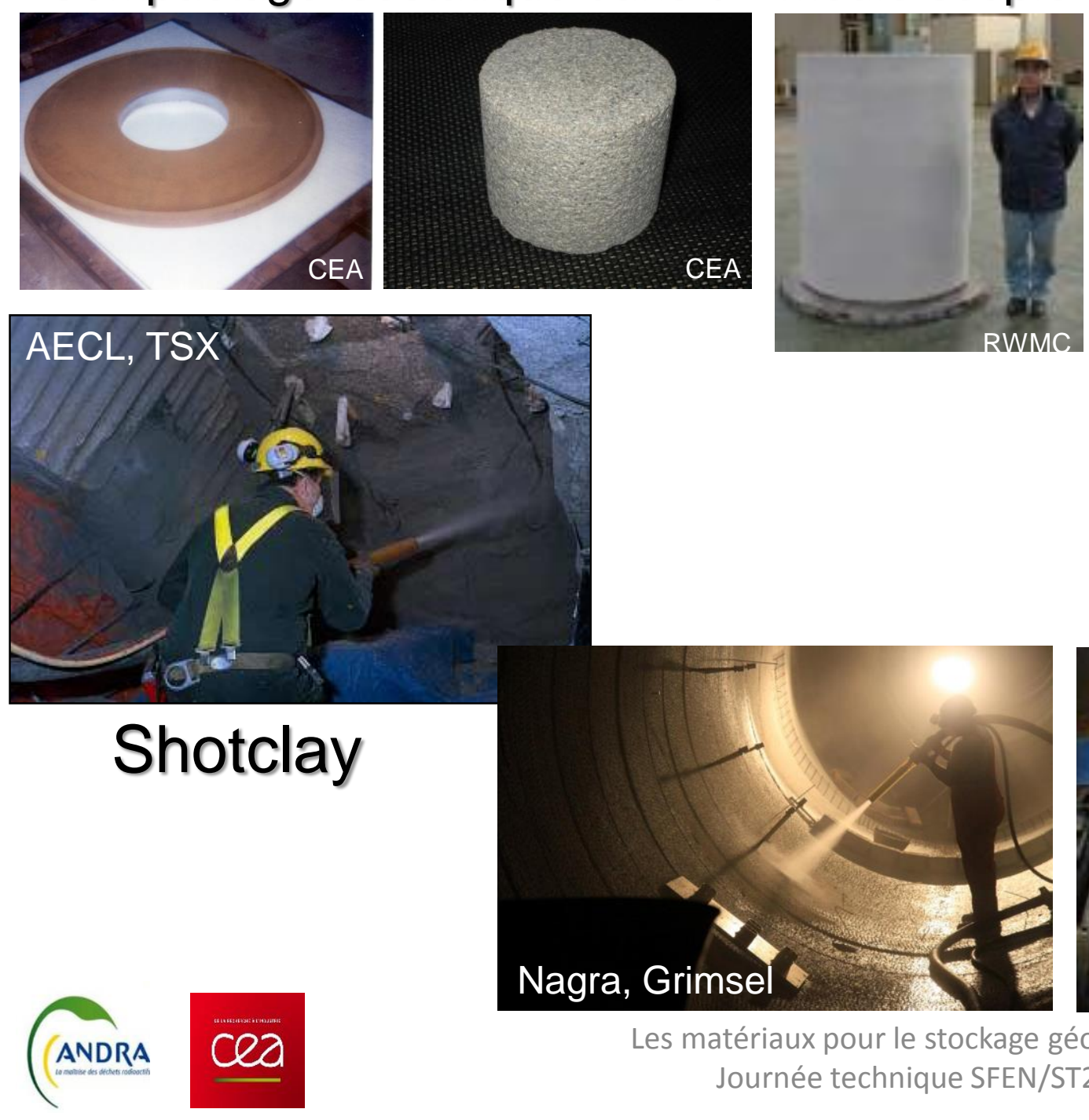

RWMC Compacted Bentonite with Center hole

Vibro-compactage

Damage
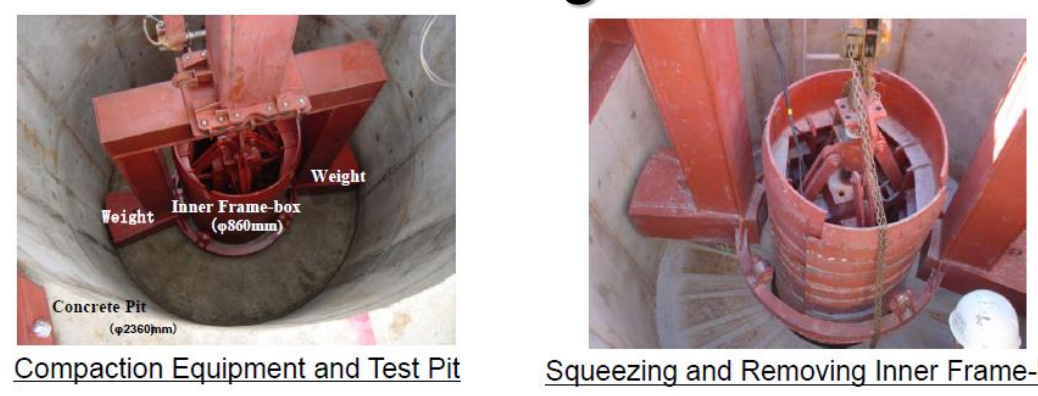

Squeezing and Removing Inner Frame-box
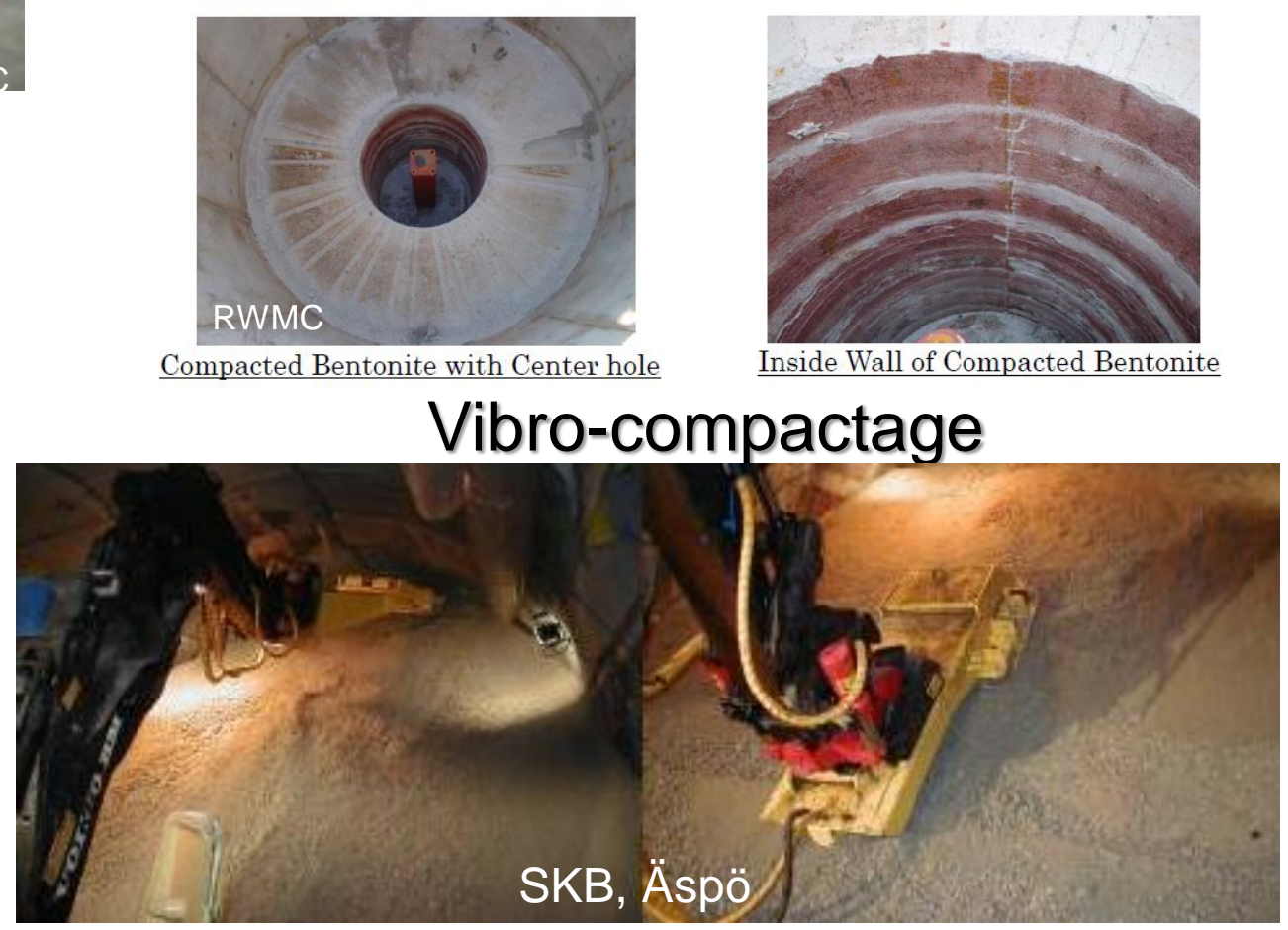


\section{Les techniques de mise en place}

Palonnier à ventouses ou

Mise en place manuelle

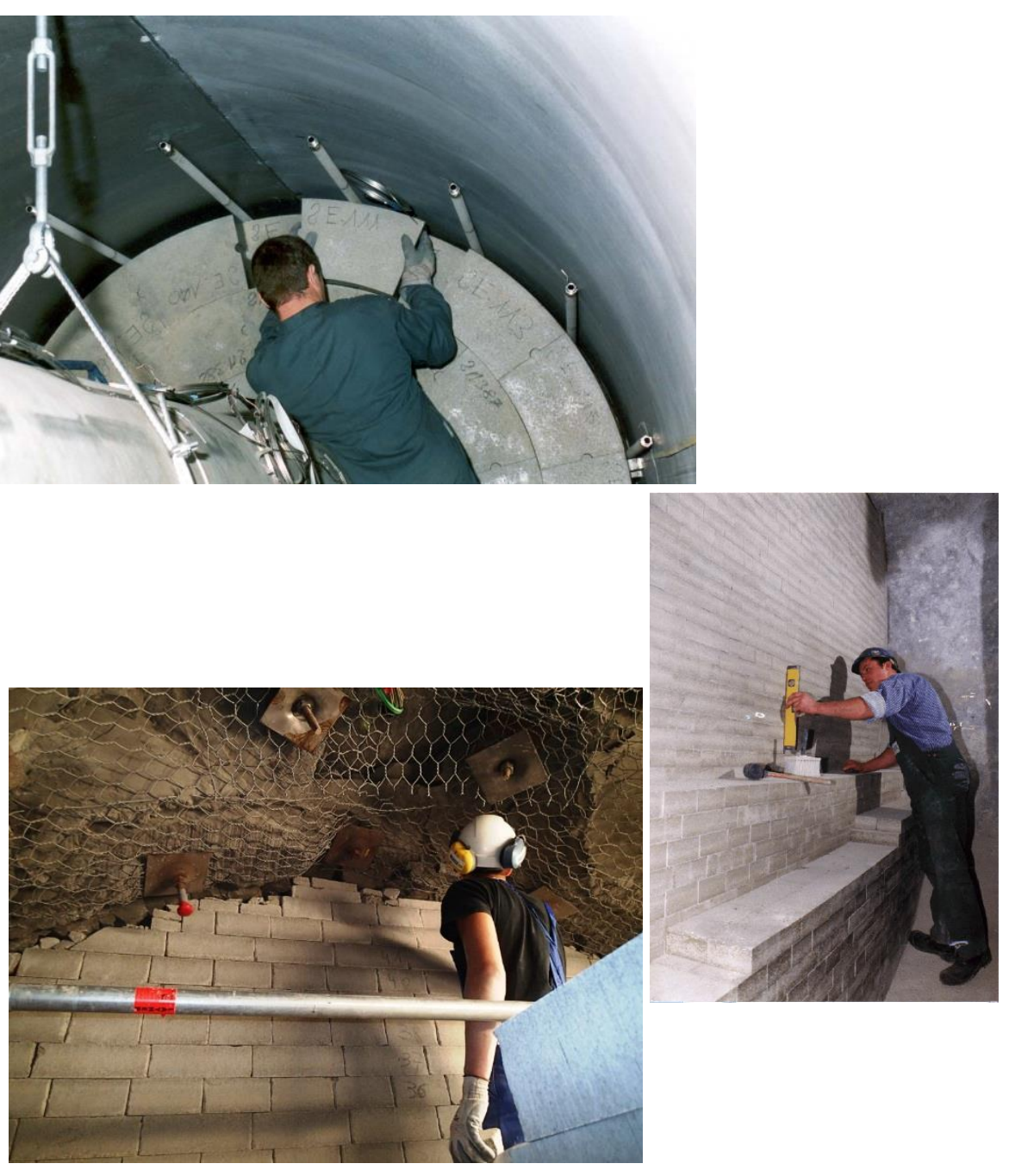

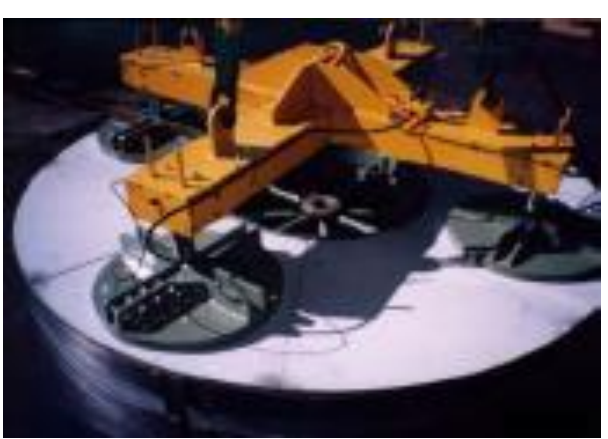

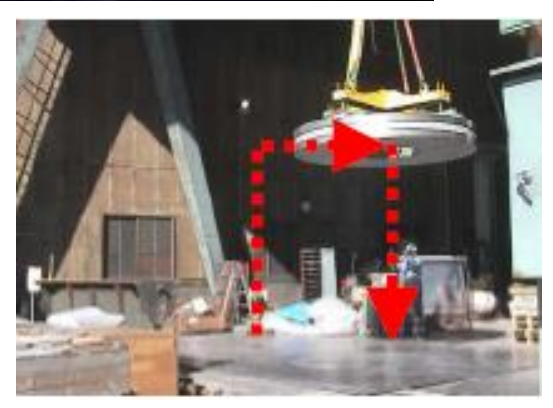

paniers métalliques

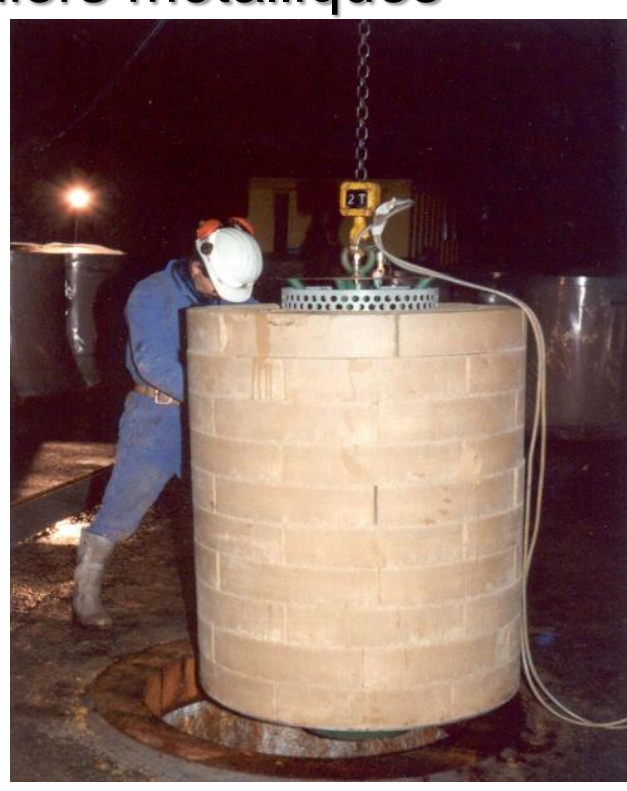

Robots avec pinces ou ventouses
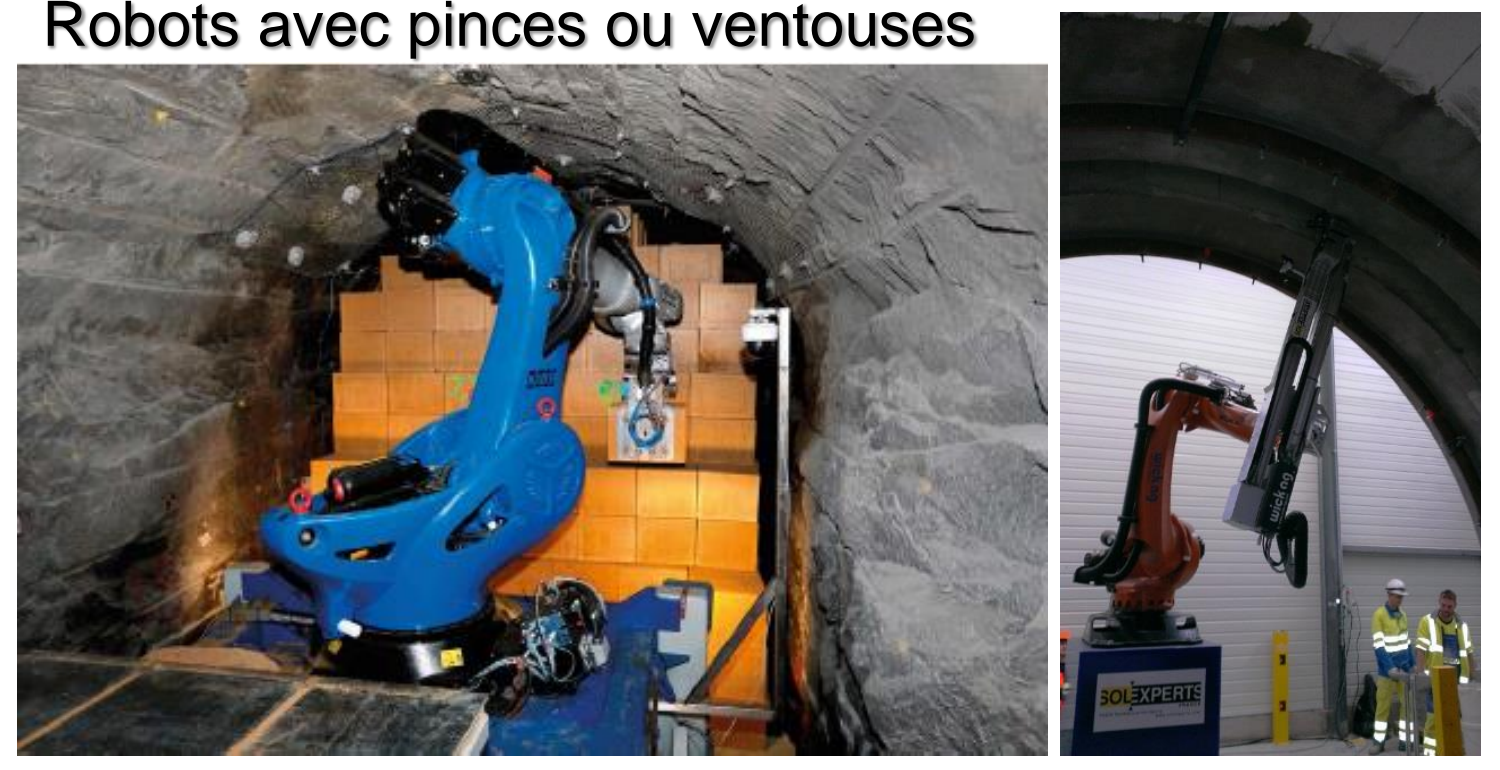


\section{Les techniques de mise en place}

Grosses pièces ou super-conteneurs Palettes à coussins d'air ou d'eau KBS-3H Deposition equipment at Äspö HRL
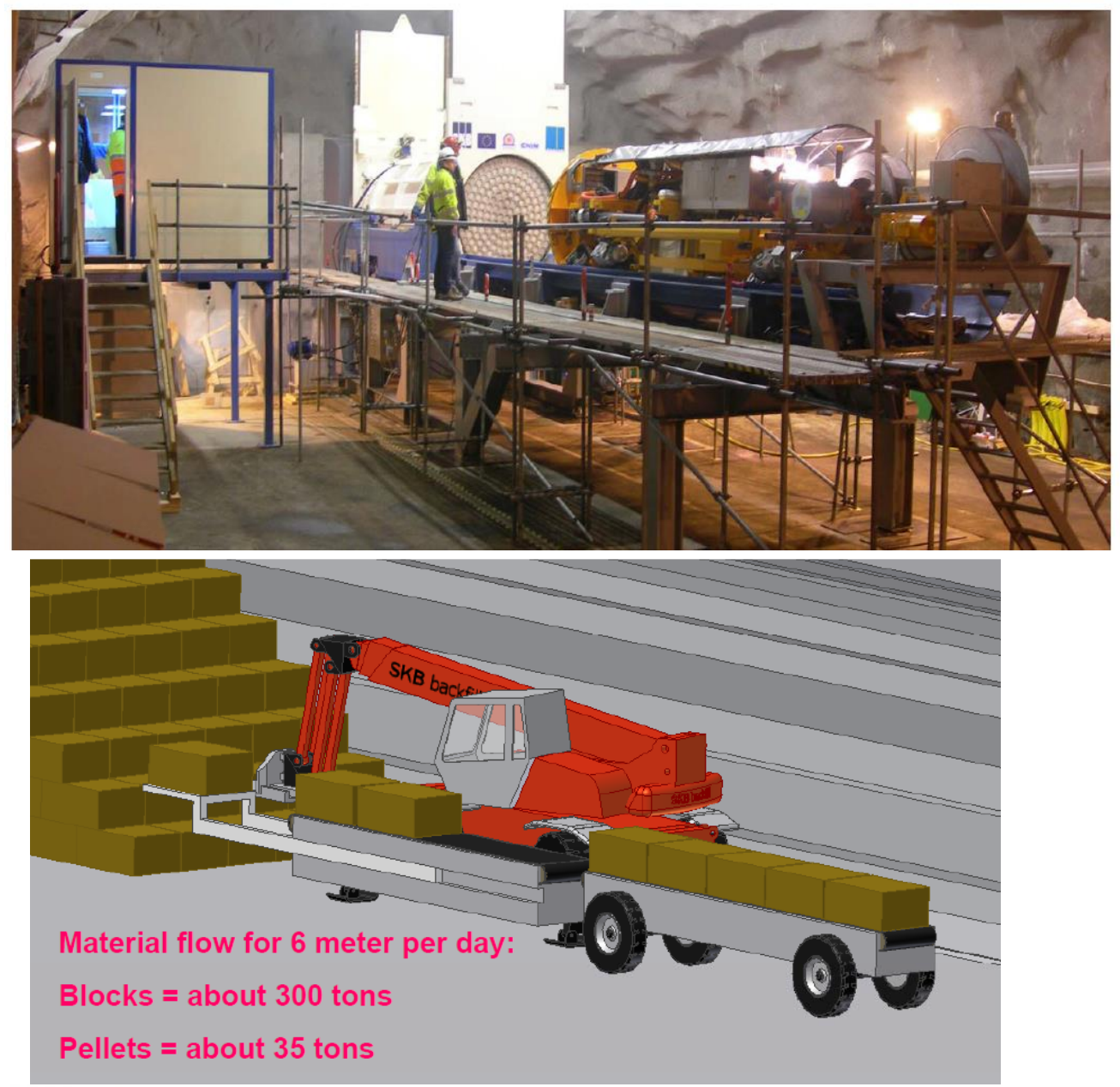

Matériaux granulaires, vis sans fin
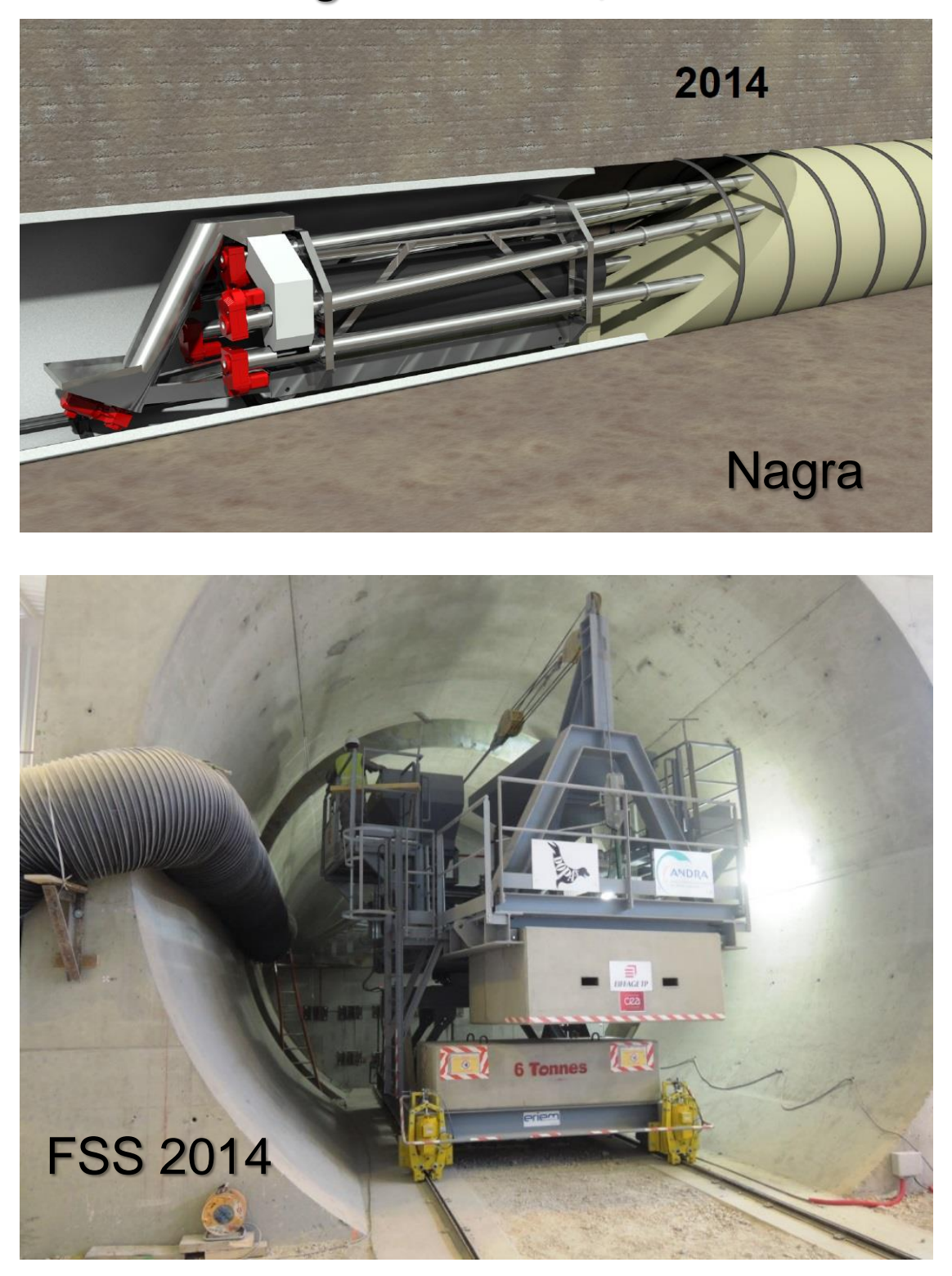


\section{L'état des connaissances \\ Les argiles gonflantes}

Les argiles gonflantes (bentonites) sont des matériaux naturels aux propriétés remarquables :

Des potentiels de sorption-désorption très élevés

Des perméabilités à l'eau et des coefficients de diffusion de la vapeur d'eau très faibles

* $\mathrm{K}<10^{-11} \mathrm{~m} / \mathrm{s}$ pour $\rho_{\mathrm{d}}>1 \mathrm{~g} / \mathrm{cm}^{3}$

$>$ Des potentiels de gonflement très élevés

$>$ Un auto-colmatage du fait du gonflement

* Fermeture des joints, des espaces inter-pellets, comblement des vides technologiques et jeux de mise en place

* Des propriétés in fine proches de celles d'un matériau massif et à même densité sèche

La bentonite du Wyoming (MX80) est souvent utilisée avec ou sans additif (sable) comme argile méthodologique de référence
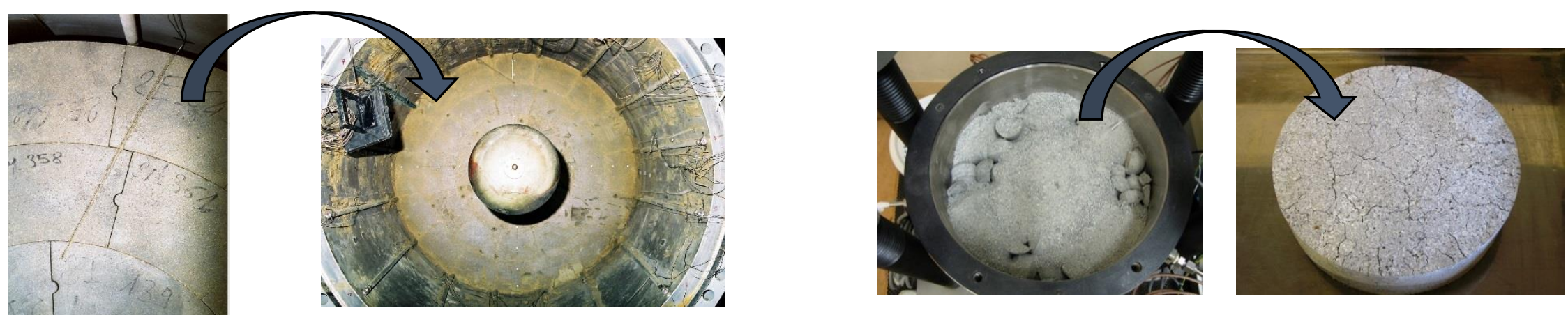


\section{L'état des connaissances}

\section{Les propriétés hydrauliques et hydromécaniques en non-saturé}

De nombreuses données disponibles et partagées par la communauté scientifique

Bonne corrélation entre expérimental et modélisation pour l'hydraulique

$>$ Transitoire HM complexe du fait des vides et des jeux technologiques

* cas des mélanges granulaires

\section{Double-porosity flow law}

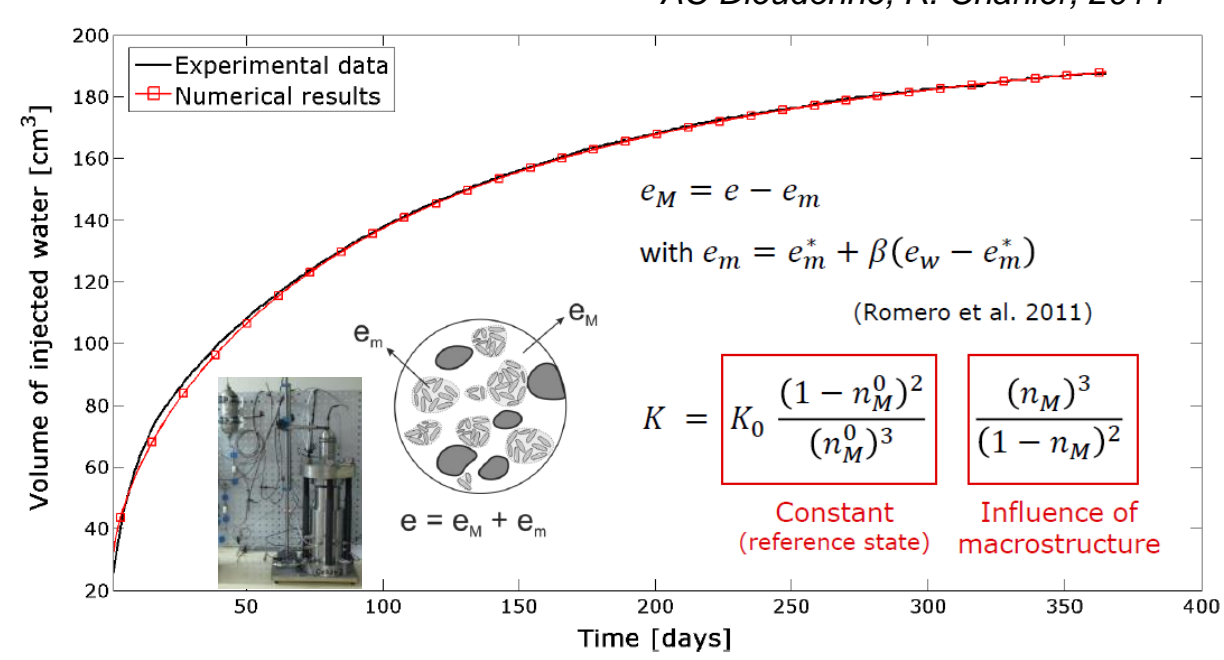

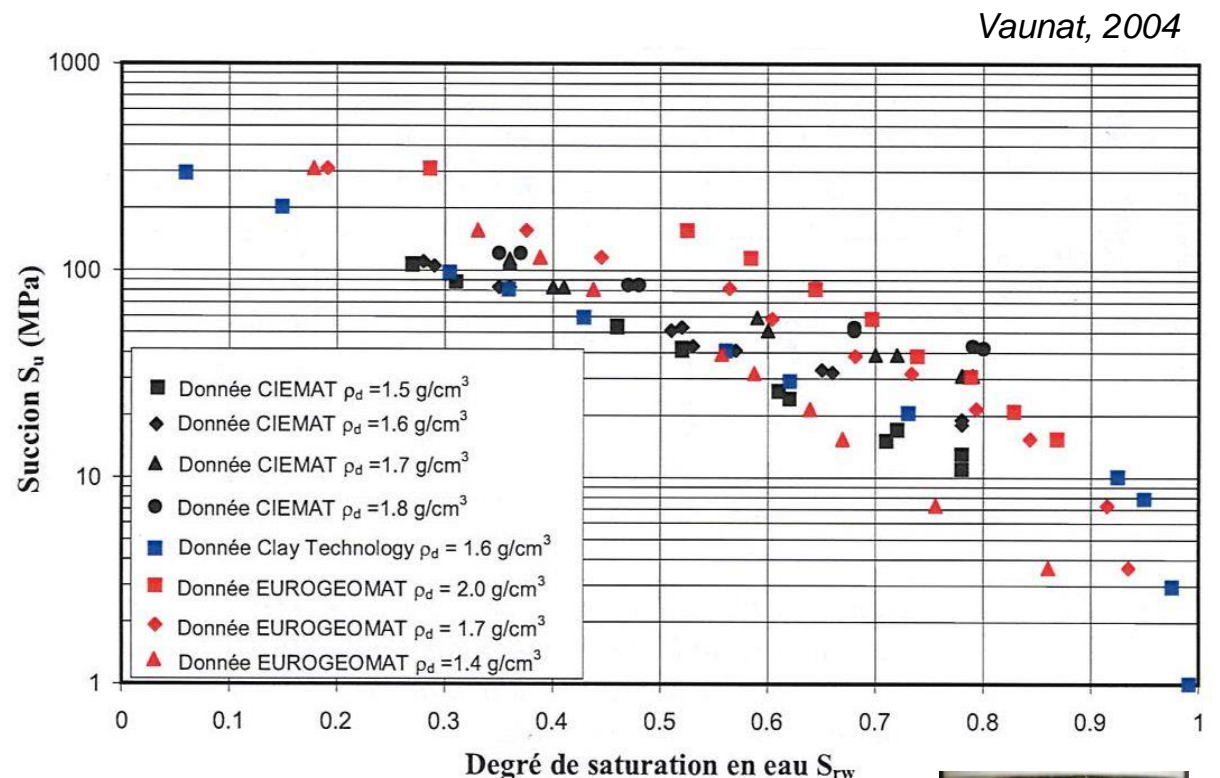

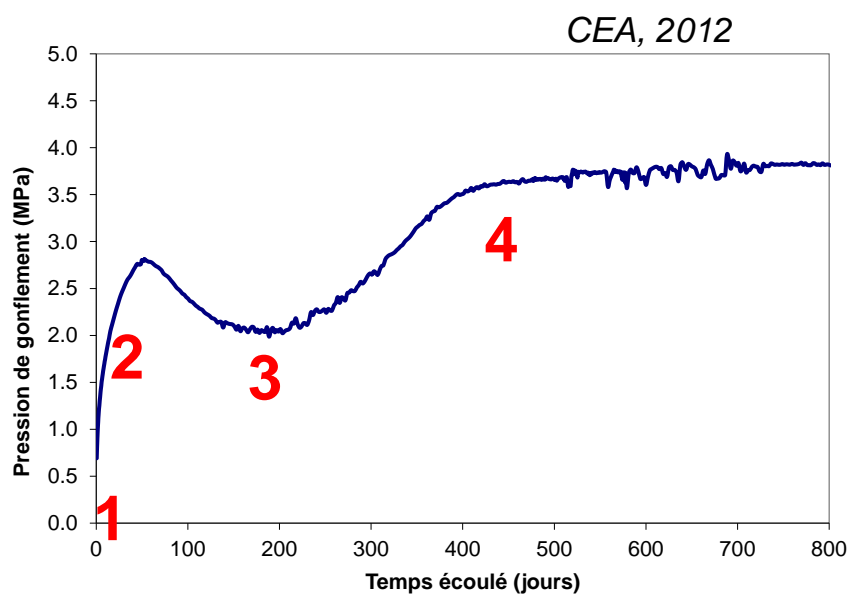

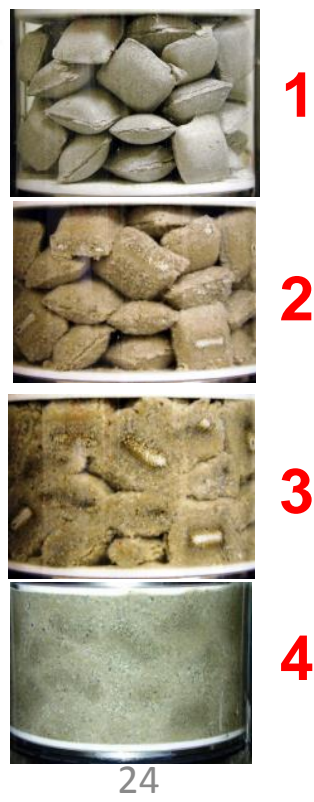




\section{L'état des connaissances}

\section{Les propriétés hydrauliques et hydromécaniques à l'état saturé}

La pression de gonflement est fonction de la densité sèche

Idem pour la perméabilité

Transfert de gaz : pression de percée $\geq$ pression de gonflement

- P. percée reproductible (dépend de $\Delta \mathrm{P}$ )

- Effet soupape

- Pas d'effet sur le comportement HM après le passage du gaz
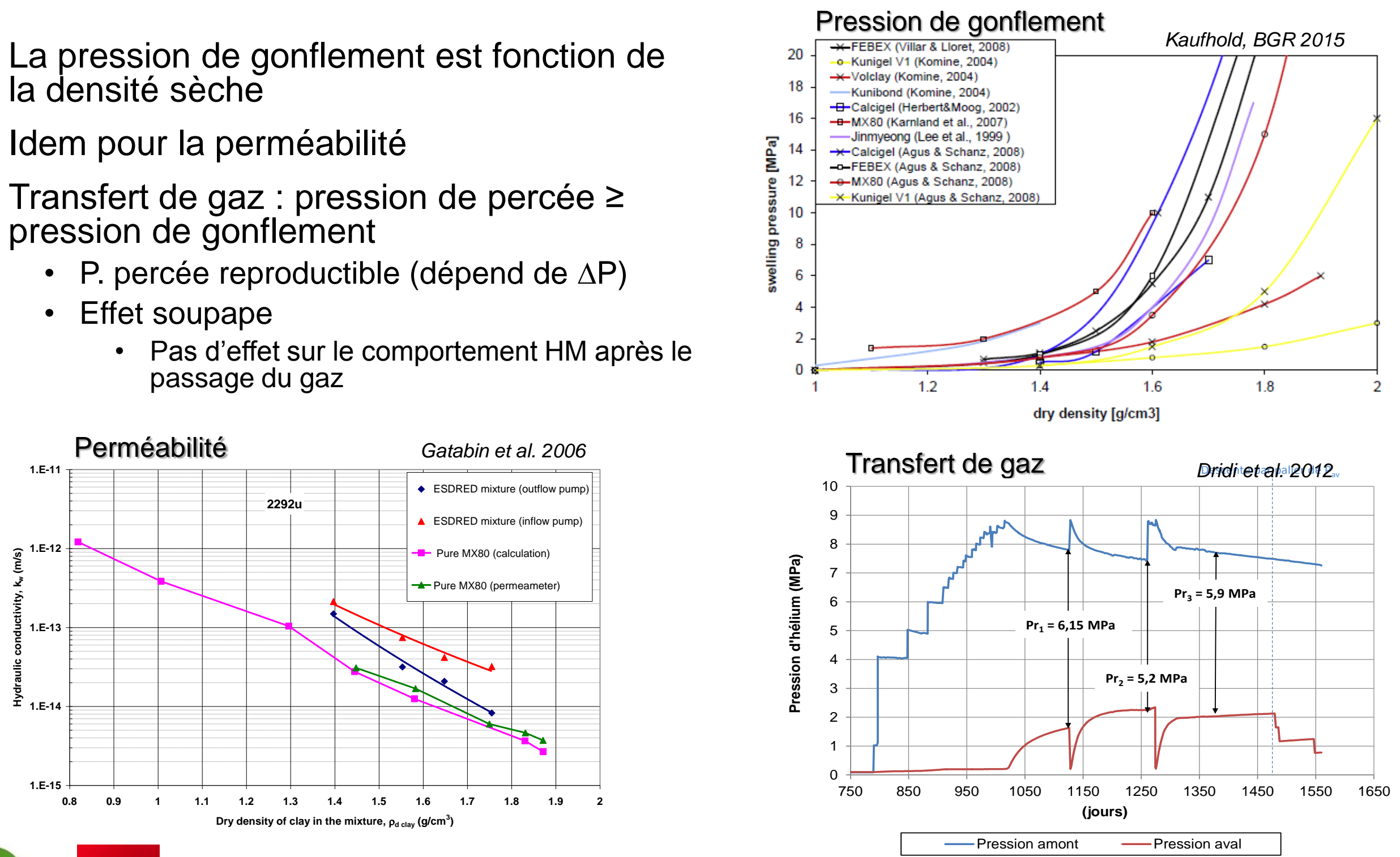

gonf 


\section{Le comportement en situation de stockage}

Transfert hydrique et transfert de gaz

Expérimentation PGZ-2 à Bure

* Scellement petit diamètre

* Noyau monolithique ou mélange pellets-poudre

* Hydratation naturelle par le massif

$\checkmark$ Sans ou avec flux de gaz

\section{Saturation du noyau et transfert du gaz} localisé aux interfaces et à l'EDZ

Rex important d'expérimentations in situ :

* TSX au Canada, Reseal en Belgique, Lasgit en Suède

Lasgit Cuss et al. 2011
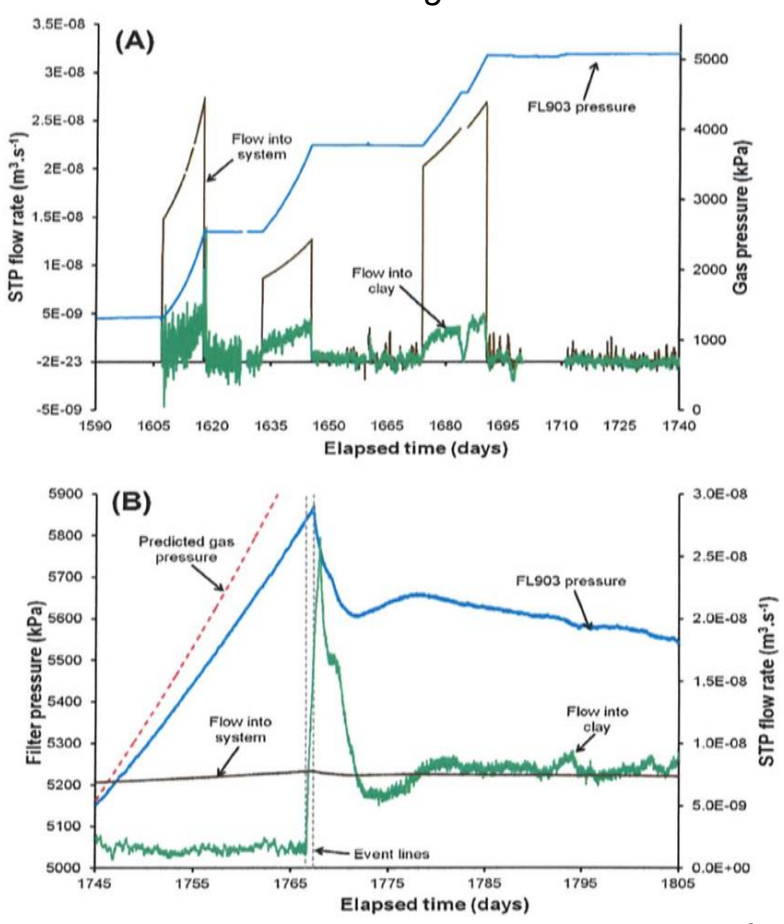

PGZ1021

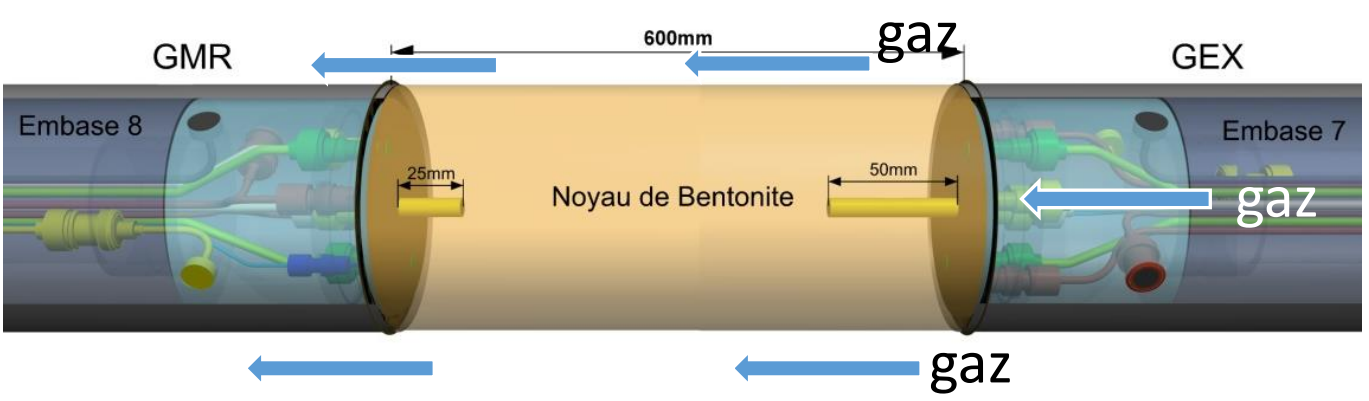

PGZ-2 De La Vaissière 2011

100

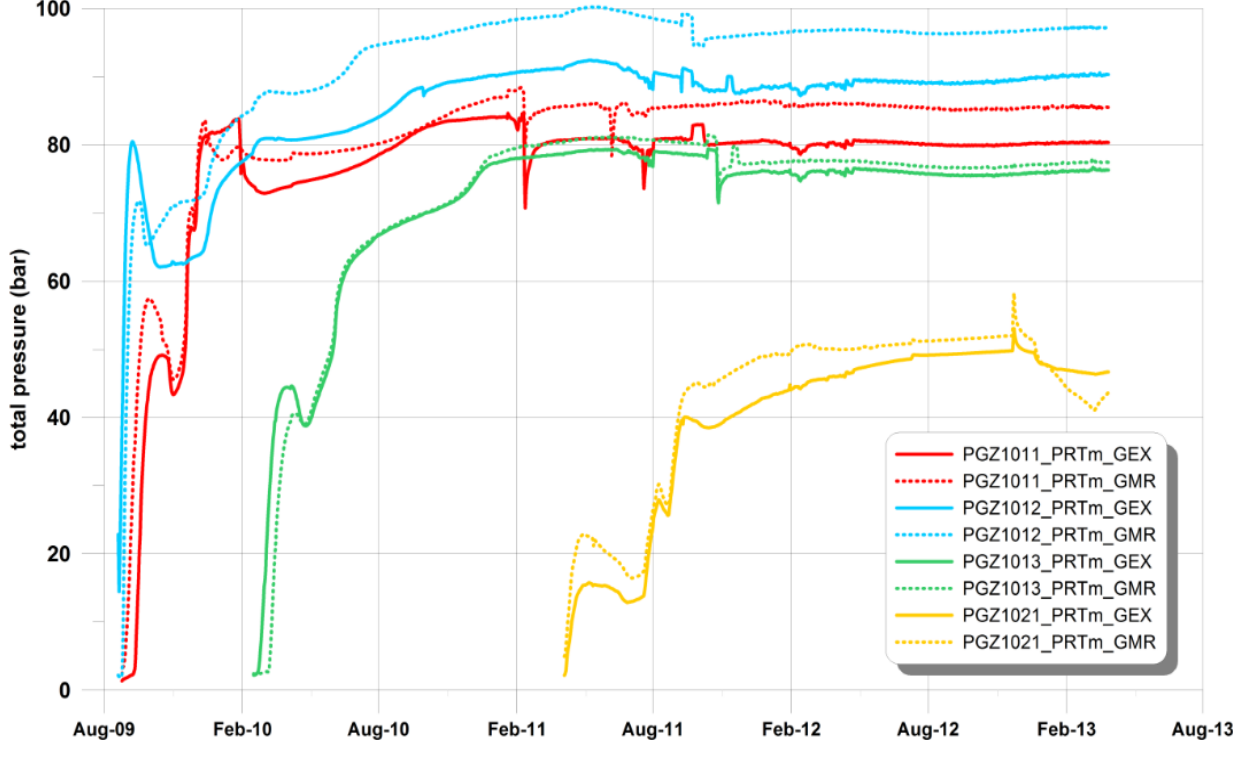




\section{Le comportement en situation de stockage}

\section{La perturbation alcaline : observations de laboratoire}

Observations sur échantillons de laboratoire

Evolutions côté argiles, suivant les bétons Bétons de pH élevé : illitisation de la smectite $\mathrm{Na}$ et de l'interstratifié I/S

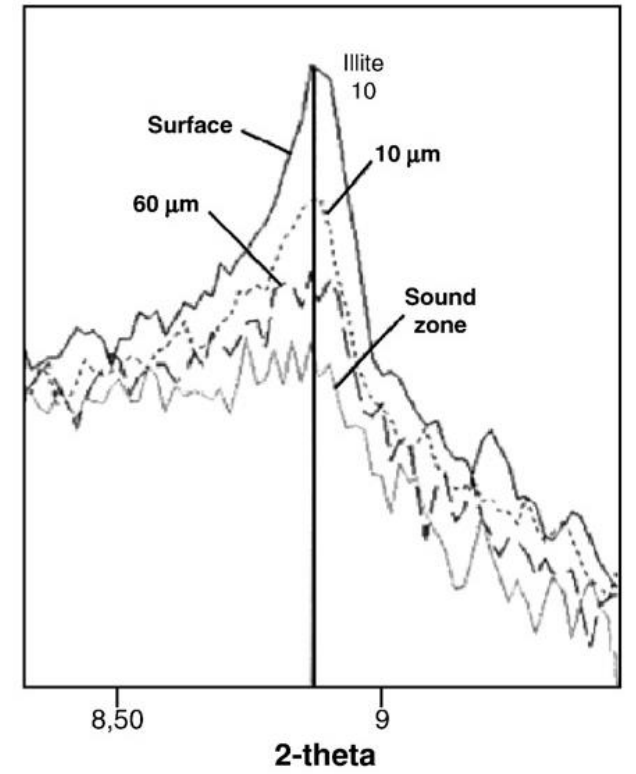

Interface pâte CEM I- argilite, 2 mois à $25^{\circ} \mathrm{C}$ (Dauzères et al, Cem.Conc.Res 2010)
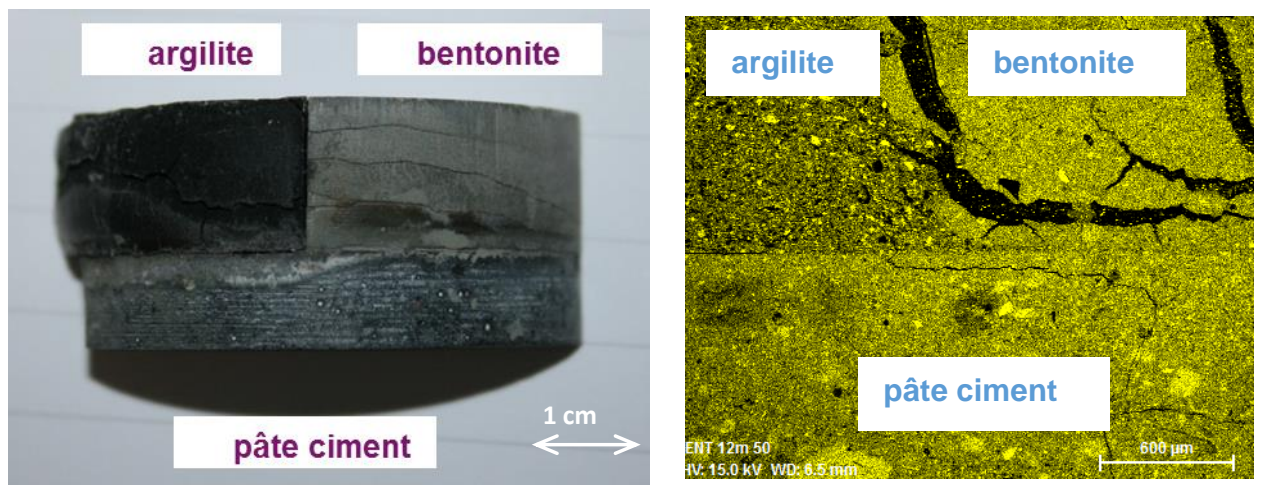

Bétons bas pH : smectite Na partiellement convertie en smectite Ca, mais peu d'impact sur le gonflement

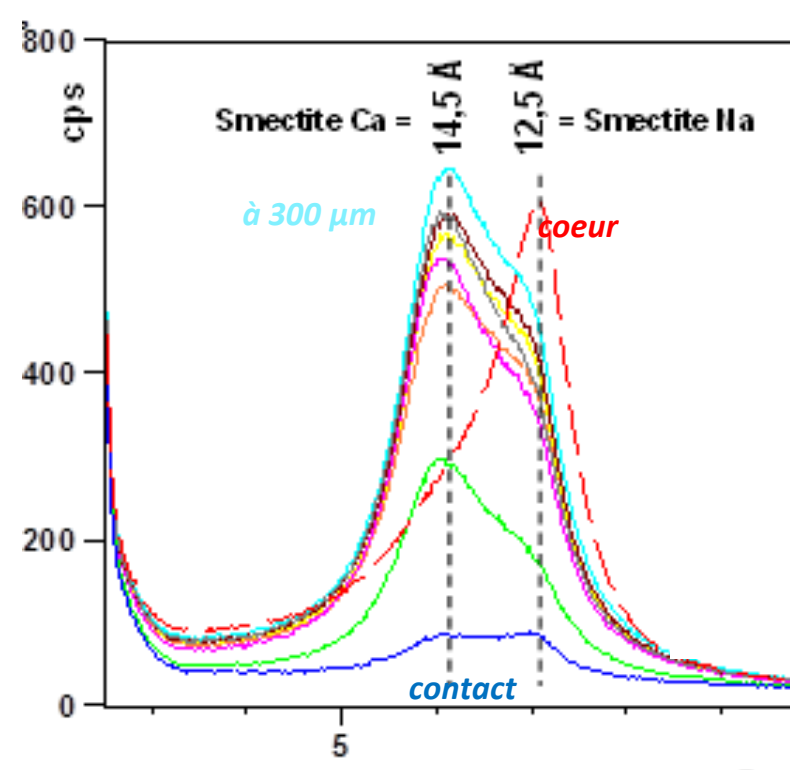

a) lames équilibrées à $54 \%$ H.R. à $25^{\circ} \mathrm{C}$

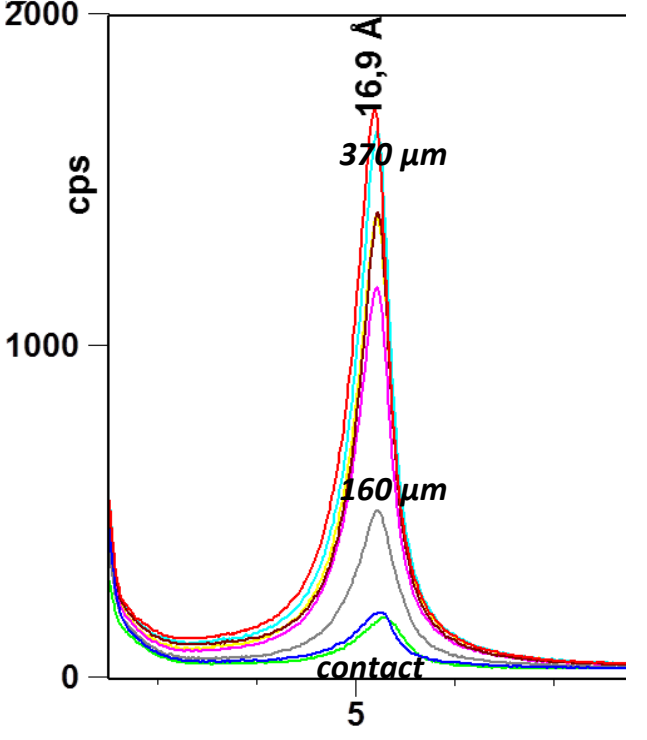

b) lames séchées à $105^{\circ} \mathrm{C}$ puis saturées à l'éthylène-glycol 


\section{Le comportement en situation de stockage la perturbation alcaline : modélisation}

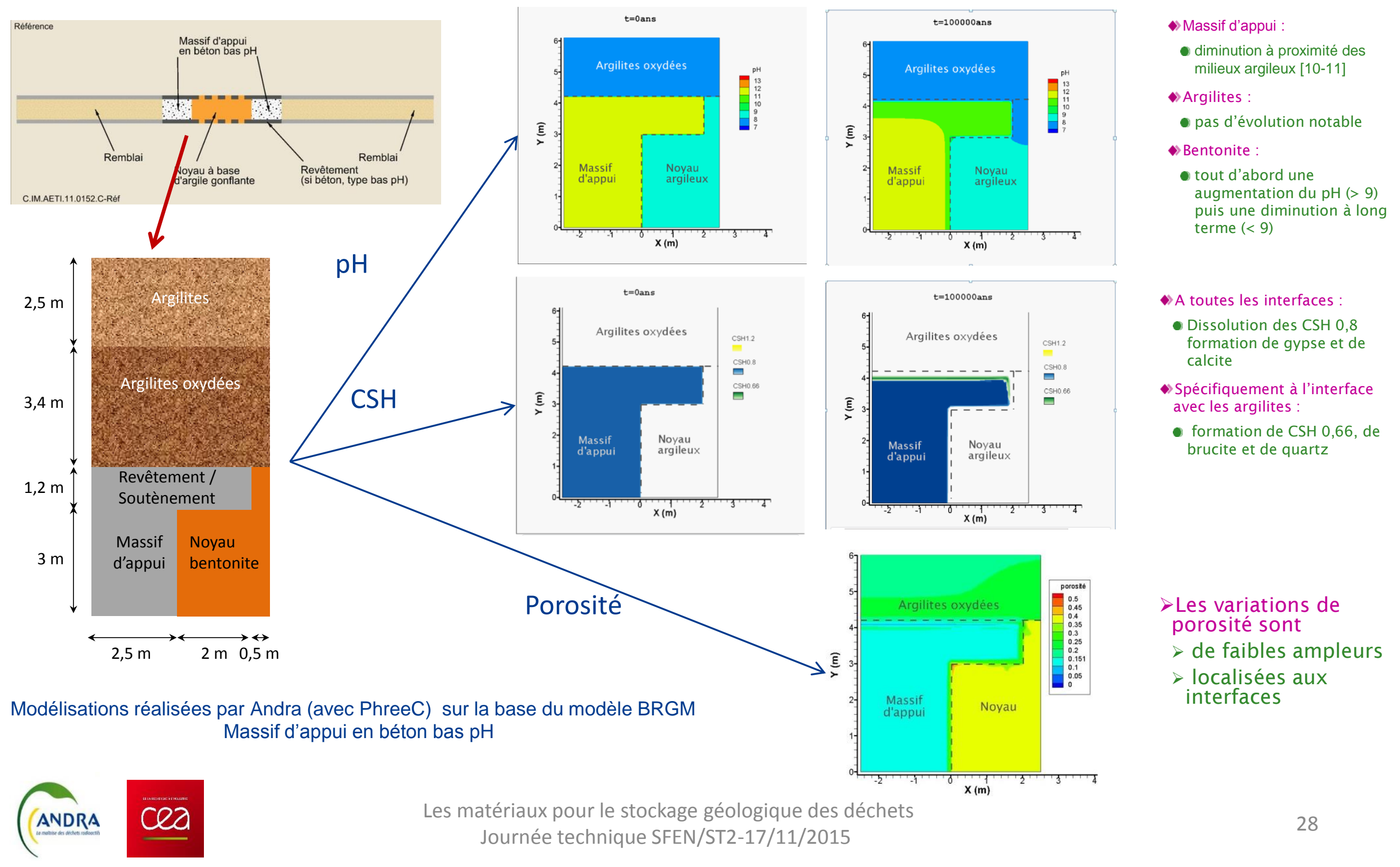




\section{Conclusion}

\section{$\square$ Bentonites}

$>$ Nombreuses données de caractérisation, méthodologies éprouvées $>$ Comportement $(\mathrm{T}) \mathrm{HM}$ bien appréhendé et prédictible

$\square$ Ouvrages de scellement

$>$ Performances requises atteintes via le gonflement de la bentonite

$>$ Transitoire de resaturation complexe mais homogénéité in fine

$>$ Temps de saturation très important

$>$ Faisabilité industrielle démontrée (incluant les appuis en béton)

\section{$\square$ Questions résiduelles}

$>$ Consolidation des connaissances et optimisation des ouvrages

* Changement d'échelle : du matériau à l'ouvrage

* Stabilité du scellement versus situation de sollicitation (T)HM-gaz 


\section{Merci de votre attention -}

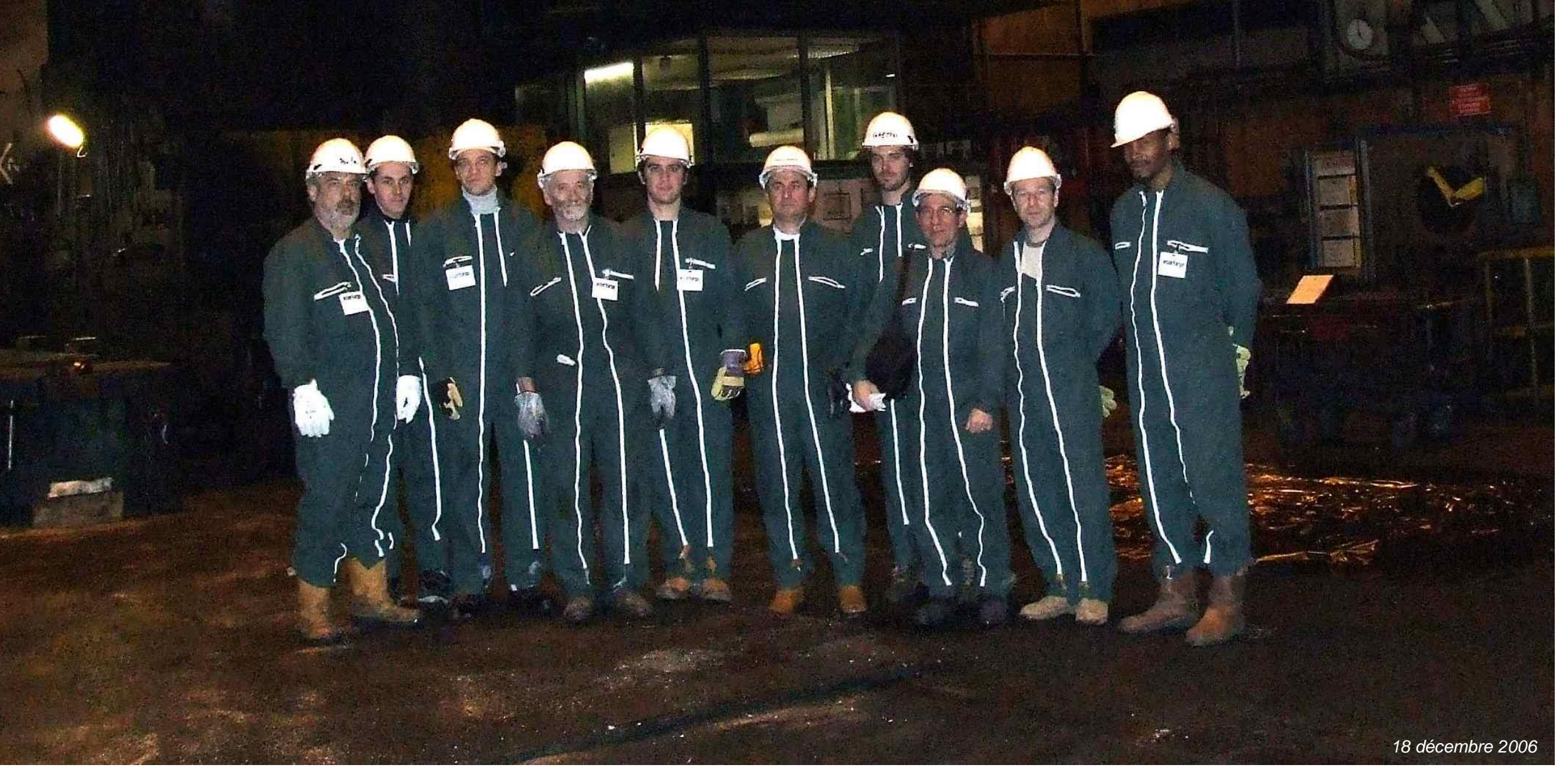

